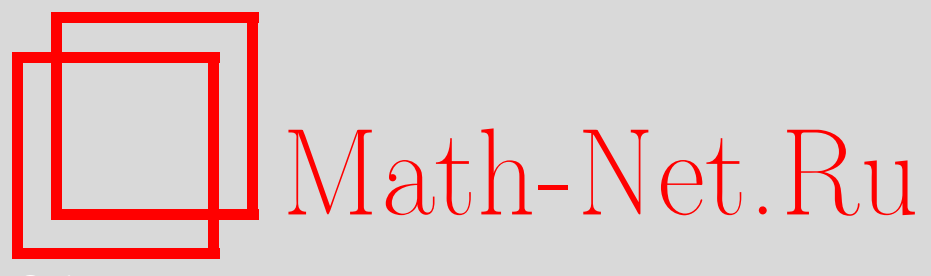

А. Ю. Колесов, Н. Х. Розов, Явление буферности в одной неклассической гиперболической краевой задаче из радиофизики, Матем. сб., 2006, том 197, номер 6, 63-96

DOI: https://doi.org/10.4213/sm1571

Использование Общероссийского математического портала Math-Net.Ru подразумевает, что вы прочитали и согласны с пользовательским соглашением http://www . mathnet.ru/rus/agreement

Параметры загрузки:

IP : 54.224 .187 .69

26 апреля 2023 г., $16: 35: 33$

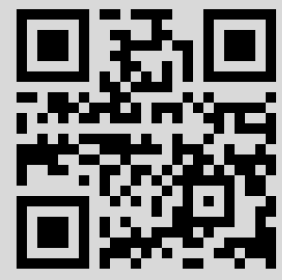


А. Ю. Колесов, Н. Х. Розов

\section{Явление буферности в одной неклассической гиперболической краевой задаче из радиофизики}

Рассматривается математическая модель $R C L$-автогенератора с отрезком соленоида в цепи обратной связи, представляющая собой следующую краевую задачу:

$$
\begin{gathered}
\frac{\partial^{2}}{\partial t^{2}}\left(u-\varkappa \frac{\partial^{2} u}{\partial x^{2}}\right)+\varepsilon \frac{\partial}{\partial t}\left(u-\varkappa \frac{\partial^{2} u}{\partial x^{2}}\right)=\frac{\partial^{2} u}{\partial x^{2}}, \\
\left.\frac{\partial u}{\partial x}\right|_{x=1}=0,\left.\quad u\right|_{x=0}+\left.\left(1+\varepsilon^{2} \gamma\right) u\right|_{x=1}-\left.u^{3}\right|_{x=1}=0,
\end{gathered}
$$

где $0<\varepsilon \ll 1$, а параметры $\varkappa, \gamma>0$ имеют порядок единицы. Для этой краевой задачи при подходящем увеличении $\gamma$ и уменьшении $\varepsilon$ устанавливается существование любого наперед заданного конечного числа устойчивых циклов (периодических по $t$ решений).

Библиография: 12 названий.

Проблема аттракторов нелинейных волновых уравнений берет свое начало с исследования автоколебаний в линейных системах телеграфных уравнений с нелинейными граничными условиями на концах конечного отрезка. Изучение такого типа краевых задач актуально в связи с тем, что они служат математическими моделями для широкого класса автогенераторов с отрезком длинной линии в цепи обратной связи. В качестве примера сошлемся на классическую работу А.А. Витта [1], являющуюся, по всей видимости, первой публикацией, посвященной настоящему вопросу. Из результатов этой статьи, а также из значительно более поздних работ [2]-[5] следует, что в случае малой диссипации в упомянутых краевых задачах наблюдается явление буферности: при подходящем выборе параметров сосуществует любое наперед заданное конечное число устойчивых периодических по времени решений.

В настоящей работе предлагается способ построения математических моделей указанных генераторов при замене в них отрезка длинной линии на отрезок прямого соленоида. Попутно поднимается вопрос о сохранении в получившихся автогенераторах явления буферности. Нетривиальность поставленной проблемы обусловлена тем, что подобная замена принципиально меняет тип уравнений в соответствующих математических моделях. Однако, как показывается ниже при анализе одного конкретного примера, в случае соленоида буферность не только сохраняется, но и наблюдается в более широком диапазоне параметров по сравнению с исходной системой.

Работа выполнена при поддержке Российского фонда фундаментальных исследований (грант № 05-01-01004).

(C) А. Ю. Колесов, Н. Х. Розов, 2006 


\section{$\S 1$. Общие свойства математической модели $R C L$-автогенератора с соленоидом в цепи обратной связи}

1.1. Физическая постановка задачи. Рассмотрим длинную линию, представляющую собой цепочку $R C L$-контуров, показанных на рис. 1 . Если связь между любыми двумя соседними ячейками отсутствует $(M=0)$, то для тока $i=i(t, x)$ и напряжения $u=u(t, x)$ в линии справедливы классические телеграфные уравнения

$$
\frac{\partial u}{\partial x}=-\frac{\partial \Phi}{\partial t}-\rho \Phi, \quad \frac{\partial i}{\partial x}=-C \frac{\partial u}{\partial t},
$$

где $\Phi=L i$ - магнитный поток, $\rho=R / L$. Однако при наличии индуктивной связи между контурами (см. рис. 1), т.е. в случае $M>0$, в уравнениях (1.1) следует положить [6], [7]

$$
\Phi=L i-M \frac{\partial^{2} i}{\partial x^{2}}
$$

В итоге после стандартного исключения из системы $(1.1),(1.2)$ переменной $i(t, x)$ и последующей замены времени $t / \sqrt{L C} \mapsto t$ для $u(t, x)$ получаем уравнение

$$
\frac{\partial^{2}}{\partial t^{2}}\left(u-\varkappa \frac{\partial^{2} u}{\partial x^{2}}\right)+\varepsilon \frac{\partial}{\partial t}\left(u-\varkappa \frac{\partial^{2} u}{\partial x^{2}}\right)=\frac{\partial^{2} u}{\partial x^{2}},
$$

в котором $\varepsilon=R \sqrt{C / L}, \varkappa=M / L$.

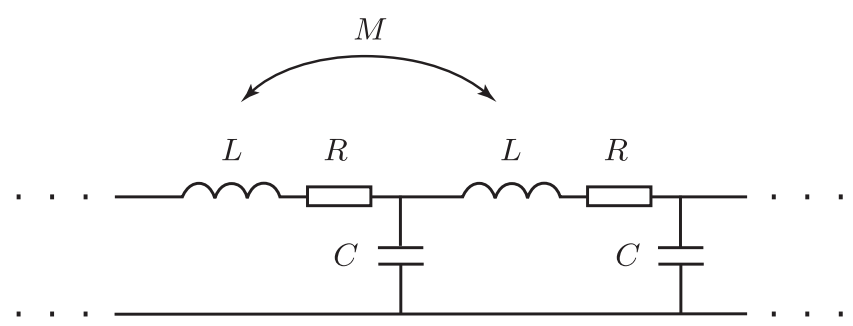

Рис. 1. Поверхность $M$

С описанной ситуацией мы сталкиваемся в случае, когда длинная линия имеет вид прямого соленоида. Действительно, каждый отдельно взятый виток соленоида можно интерпретировать как элементарный $R C L$-контур. Тогда при естественном предположении о наличии индуктивной связи между соседними витками приходим к уравнению (1.3).

В этой работе объектом исследования является автогенератор, блок-схема которого представлена на рис. 2, а). Предполагаем, что в цепь обратной связи этого генератора включен отрезок $0 \leqslant x \leqslant 1$ прямого соленоида с равномерно распределенными индуктивностью $L$, активным сопротивлением $R$ и емкостью $C$. Считаем, далее, что вольт-амперная характеристика его нелинейного элемента (в простейшем случае - классической лампы) имеет вид, изображенный на рис. 2,б). Тогда, опираясь на методику работы [8], можно показать, 
что при дополнительном предположении об идеальности усилителя математической моделью описанного генератора служит уравнение (1.3), рассматриваемое на отрезке $0 \leqslant x \leqslant 1$ и дополненное граничными условиями

$$
\left.\frac{\partial u}{\partial x}\right|_{x=1}=0,\left.\quad u\right|_{x=0}+\left.K u\right|_{x=1}-\left.u^{3}\right|_{x=1}=0 .
$$

Здесь $u$ - нормированная переменная составляющая напряжения в линии, $K>0$ - коэффициент усиления, а функция $K u-u^{3}$ представляет собой аппроксимацию нелинейной характеристики в окрестности рабочей точки $A$ (см. рис. 2, б)).

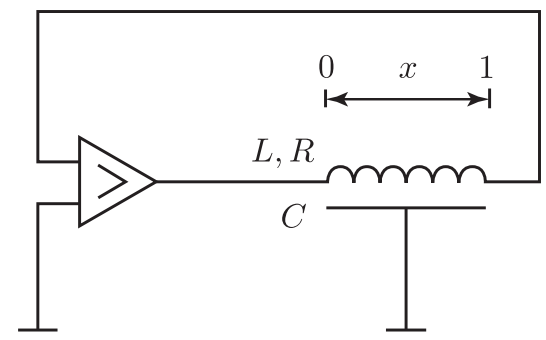

a)

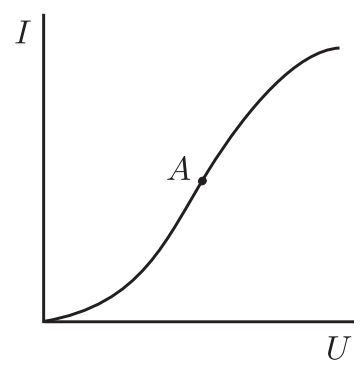

б)

Рис. 2

1.2. Разрешимость задачи Коши. Начнем с вопроса о разрешимости краевой задачи (1.3), (1.4) в подходящем фазовом пространстве. С этой целью введем в рассмотрение вещественное гильбертово пространство $E$, состоящее из функций $u(x) \in \mathrm{W}_{2}^{2}(0,1)$, удовлетворяющих граничным условиям

$$
\left.\frac{d u}{d x}\right|_{x=1}=0,\left.\quad u\right|_{x=0}+\left.u\right|_{x=1}=0 .
$$

Скалярное произведение для любых функций $u_{1}, u_{2} \in E$ определим равенством

$$
\left(u_{1}, u_{2}\right)_{E}=\int_{0}^{1} u_{1}^{\prime \prime}(x) u_{2}^{\prime \prime}(x) d x .
$$

Далее, нам потребуется дифференциальный оператор

$$
L=-\frac{d^{2}}{d x^{2}}: E \subset \mathrm{L}_{2}(0,1) \rightarrow \mathrm{L}_{2}(0,1),
$$

обладающий следующими свойствами. Во-первых, все его собственные значения $\lambda_{n}=\omega_{n}^{2}, \omega_{n}=\pi(2 n-1), n=1,2, \ldots$, двукратны, так как каждому из них наряду с собственной функцией $\cos \omega_{n} x$ отвечает одна присоединенная функция $\left(2 \omega_{n}\right)^{-1}(1-x) \sin \omega_{n} x$. Во-вторых, система функций

$$
e_{n, 1}(x)=\cos \omega_{n} x, \quad e_{n, 2}(x)=(x-1) \sin \omega_{n} x, \quad n=1,2, \ldots,
$$

образует базис Рисса в пространстве $\mathrm{L}_{2}(0,1)$, а после деления на $\omega_{n}^{2}$ будет базисом в $E$ (доказательство этого факта приведено, например, в [5]). 
Из всего вышесказанного следует, что при любом $\varkappa>0$ оператор $I+\varkappa L$, где $I$ - единичный оператор, имеет ограниченный обратный $(I+\varkappa L)^{-1}$, действующий из $\mathrm{L}_{2}(0,1)$ в $E$ по формуле

$$
(I+\varkappa L)^{-1} u(x)=\sum_{n=1}^{\infty} \frac{1}{1+\varkappa \omega_{n}^{2}}\left[\alpha_{n} e_{n, 1}(x)+\beta_{n}\left(e_{n, 2}(x)+\frac{2 \varkappa \omega_{n}}{1+\varkappa \omega_{n}^{2}} e_{n, 1}(x)\right)\right] .
$$

Здесь $\alpha_{n}, \beta_{n}, n \geqslant 1,-$ коэффициенты разложения

$$
u(x)=\sum_{n=1}^{\infty}\left(\alpha_{n} \cos \omega_{n} x+\beta_{n}(x-1) \sin \omega_{n} x\right)
$$

произвольной функции $u(x) \in \mathrm{L}_{2}(0,1)$ по базису (1.6).

В формулируемом ниже утверждении через $B(r)$ обозначен замкнутый шар пространства $\mathrm{W}_{2}^{2}(0,1)$ с центром в нуле радиуса $r$, а в качестве фазового пространства (пространства начальных условий $(u, \partial u / \partial t))$ краевой задачи (1.3), (1.4) взято нелинейное многообразие

$$
\begin{aligned}
\Sigma=\{ & \left(u_{0}(x), u_{1}(x)\right): u_{0}, u_{1} \in \mathrm{W}_{2}^{2}(0,1), u_{0}^{\prime}(1)=u_{1}^{\prime}(1)=0, \\
& \left.u_{0}(0)+K u_{0}(1)-u_{0}^{3}(1)=0, u_{1}(0)+\left(K-3 u_{0}^{2}(1)\right) u_{1}(1)=0\right\} .
\end{aligned}
$$

Теорема 1.1. Пусть в краевой задаче (1.3), (1.4) параметры $\varepsilon, \varkappa>0$ и коэффициент $K>0$ произвольно фиксированы. Тогда по любому $T>0$ можно указать такое достаточно малое $r=r(T)>0$, что при $t \in[-T, T]$ существует единственное решение

$$
u=u(t, x):\left.u\right|_{t=0}=u_{0}(x),\left.\quad \frac{\partial u}{\partial t}\right|_{t=0}=u_{1}(x),
$$

данной задачи с произвольными начальными условиями

$$
\left(u_{0}, u_{1}\right) \in \Sigma \cap B(r) \times B(r),
$$

обладающее следующими свойствами. Во-первых, функиии и, $\partial u / \partial t, \partial^{2} u / \partial t^{2}$ непрерывны по $t \in[-T, T]$ в метрике пространства $\mathrm{W}_{2}^{2}(0,1)$, а функции $h_{j}$, $\partial h_{j} / \partial t, \partial^{2} h_{j} / \partial t^{2}$, где $h_{j}=\partial^{j} u / \partial x^{j}, j=1,2$, обладают тем же свойством в метрике $\mathrm{L}_{2}(0,1)$. Во-вторых, справедливо включение

$$
\left\{\left(u(t, x), \frac{\partial u}{\partial t}(t, x)\right): t \in[-T, T]\right\} \subset \Sigma,
$$

а самому уравнению (1.3) решение (1.10) при каждом $t$ удовлетворяет в смысле $\mathrm{L}_{2}(0,1)$.

ДокАЗАТЕльство. Основная идея обоснования заключается в сведении исходной краевой задачи (1.3), (1.4) к абстрактному нелинейному дифференциальному уравнению в пространстве $E \times E$ с ограниченными операторными коэффициентами. Для построения замены переменных, осуществляющей указанное сведение, подставим в граничные условия (1.4) соотношение

$$
u=v+l(v),
$$


где $v$ - произвольная функция из пространства $E$, а $l(\cdot): E \rightarrow \mathbb{R}$ - некоторый (пока неизвестный) нелинейный функционал. В результате для нахождения $l(v)$ получаем уравнение

$$
(1+K) l(v)+\left.(K-1) v\right|_{x=1}-\left(\left.v\right|_{x=1}+l(v)\right)^{3}=0 .
$$

Введем в рассмотрение вспомогательное уравнение

$$
(1+K) w+(K-1) z-(z+w)^{3}=0
$$

на плоскости $(z, w) \in \mathbb{R}^{2}$ и заметим, что в точке $z=0, w=0$ к нему применима теорема о неявной функции по переменной $w$. Таким образом, из (1.14) сначала находим функцию

$$
w=w_{0}(z), \quad w_{0}(0)=0, \quad w_{0}^{\prime}(0)=\frac{1-K}{1+K},
$$

а затем полагаем

$$
l(v)=w_{0}\left(\left.v\right|_{x=1}\right) .
$$

Нетрудно увидеть, что получившийся на этом пути функционал (1.16) удовлетворяет требуемому равенству (1.13). Кроме того, он определен и аналитичен по $v$ в некоторой достаточно малой окрестности нуля пространства $E$, поскольку аналогичными свойствами обладает функция (1.15) в окрестности точки $z=0$.

На следующем этапе доказательства подставим формулу (1.12) в уравнение (1.3). В результате оно преобразуется к виду

$$
(I+\varkappa L)\left(\frac{\partial^{2} v}{\partial t^{2}}+\varepsilon \frac{\partial v}{\partial t}\right)+\left(\frac{\partial^{2}}{\partial t^{2}}+\varepsilon \frac{\partial}{\partial t}\right) l(v)=-L v,
$$

где $L$ - оператор (1.5). Применяя, далее, к левой и правой частям равенства (1.17) оператор $(I+\varkappa L)^{-1}($ см. $(1.7),(1.8))$, окончательно получаем

$$
\frac{\partial^{2} v}{\partial t^{2}}+\varepsilon \frac{\partial v}{\partial t}+f(x)\left(\frac{\partial^{2}}{\partial t^{2}}+\varepsilon \frac{\partial}{\partial t}\right) l(v)=-(I+\varkappa L)^{-1} L v,
$$

где

$$
f(x)=1-\frac{2 \operatorname{ch}[(x-1) / \sqrt{\varkappa}]}{1+\operatorname{ch}(1 / \sqrt{\varkappa})} \in E,
$$

а оператор $(I+\varkappa L)^{-1} L$ действует из $E$ в $E$ и ограничен.

Обращаем внимание, что (1.18) - это и есть искомое уравнение в пространстве $E$ с ограниченными операторными коэффициентами. Поэтому дополним его произвольными начальными условиями

$$
\left.v\right|_{t=0}=v_{0},\left.\quad \frac{\partial v}{\partial t}\right|_{t=0}=v_{1}, \quad\left(v_{0}, v_{1}\right) \in S\left(r_{0}\right) \times S\left(r_{0}\right),
$$

где $S\left(r_{0}\right)$ - замкнутый шар в $E$ с центром в нуле достаточно малого радиуса $r_{0}>0$.

Требование малости $r_{0}$ в (1.20) обусловлено прежде всего тем обстоятельством, что функционал (1.16) определен лишь локально. Другая причина, по 
которой уравнение (1.18) приходится рассматривать только в достаточно малой окрестности нуля, заключается в том, что оно не разрешено относительно старшей производной по времени. Действительно, перед $\partial^{2} / \partial t^{2}$ стоит линейный оператор $C(v)$, действующий на элементах $h(x) \in E$ по правилу

$$
C(v) h=h(x)+\left.f(x) w_{0}^{\prime}\left(\left.v\right|_{x=1}\right) h\right|_{x=1},
$$

где $w_{0}(z), f(x)$ - функции $(1.15),(1.19)$. Однако при всех достаточно малых $v \in E$ оператор (1.21) непрерывно обратим, причем для соответствующего обратного оператора справедливо равенство

$$
C^{-1}(v) h=h(x)+\left.f(x) w_{0}^{\prime}\left(\left.v\right|_{x=1}\right) c h\right|_{x=1},
$$

где

$$
c=-\frac{1}{1+w_{0}^{\prime}\left(\left.v\right|_{x=1}\right) f(1)} .
$$

Действительно, в силу очевидных оценок $|(K-1) /(K+1)|<1,0<f(1)<1$ и свойств функции (1.15) знаменатель в (1.23) отличен от нуля при $v=0$, а значит, и при всех достаточно малых $v \in E$.

Суммируя изложенные факты, приходим к выводу о существовании для любого фиксированного $T>0$ достаточно малого числа $r_{0}=r_{0}(T)>0$ со следующими свойствами. Во-первых, при всех $v \in S\left(r_{0}\right)$ оператор (1.21) является обратимым (см. (1.22), (1.23)), а значит, уравнение (1.18) разрешается относительно $\partial^{2} / \partial t^{2}$. Во-вторых, дополним уравнение, получающееся из (1.18) после указанного разрешения, начальными условиями (1.20). В итоге получаем задачу Коши, к которой применимы результаты монографии [9]. Из этих результатов (см. [9; гл. $1, \S 3])$ вытекают существование и единственность (при надлежащем уменьшении $r_{0}$ в $\left.(1.20)\right)$ решения $v=v(t, x)$ рассматриваемой задачи на отрезке $t \in[-T, T]$. Отметим еще, что функция $v(t, x)$ является бесконечно дифференцируемой по $t$ в метрике пространства $E$, что следует из ограниченности входящих в (1.18) операторов и из аналогичных свойств гладкости функционала (1.16).

Заключительный этап обоснования теоремы состоит в перенесении результатов, полученных для задачи (1.18), (1.20), на исходную краевую задачу (1.3), (1.4). Для этого нам потребуются равенства

$$
u_{0}=v_{0}+l\left(v_{0}\right), \quad u_{1}=v_{1}+l^{\prime}\left(v_{0}\right) v_{1},
$$

где $l^{\prime}$ - производная Фреше функционала (1.16), связывающие начальные условия из (1.20) и (1.10). Убедимся прежде всего, что эти равенства задают диффеоморфизм между диском (1.11), лежащим на многообразии (1.9), и некоторой окрестностью нуля пространства $E \times E$ (при условии, конечно, что радиус $r$ в (1.11) достаточно мал).

Обратимся сначала к первому уравнению из (1.24) и применим к нему теорему о неявном отображении в точке $u_{0}=0, v_{0}=0$. Проверка выполнения условий этой теоремы сводится к доказательству непрерывной обратимости линейного оператора

$$
C h(x)=h(x)-\frac{K-1}{K+1} h(1)
$$


действующего из $E$ в пространство $\stackrel{\circ}{\mathrm{W}}_{2}^{2}(0,1)$, состоящее из функций $u(x)$ класса $\mathrm{W}_{2}^{2}$, удовлетворяющих граничным условиям

$$
\left.\frac{d u}{d x}\right|_{x=1}=0,\left.\quad u\right|_{x=0}+\left.K u\right|_{x=1}=0 .
$$

Несложный подсчет показывает, что оператор (1.25) обладает нужным свойством, а соответствующий ему обратный оператор задается аналогичным (1.22) равенством

$$
C^{-1} u(x)=u(x)+\frac{K-1}{2} u(1) .
$$

Что же касается второго уравнения из (1.24), то его анализ идентичен описанному выше.

Факт взаимной однозначности отображения (1.24) в окрестности точки $\left(v_{0}, v_{1}\right)=(0,0)$ позволяет, в свою очередь, однозначно определить интересующее нас решение (1.10) задачи (1.3), (1.4) по формулам

$$
u=v+l(v), \quad \frac{\partial u}{\partial t}=\frac{\partial v}{\partial t}+l^{\prime}(v) \frac{\partial v}{\partial t},
$$

где $v$ - соответствующее решение задачи $(1.18),(1.20)$. Из этих формул, а также из установленных ранее свойств $v(t, x)$ следует, что функция $u(t, x)$ заведомо удовлетворяет всем требуемым в теореме условиям гладкости. Более того, $u \in C^{\infty}$ по переменной $t$ в метрике $\mathrm{W}_{2}^{2}$, а для $\partial u / \partial x, \partial^{2} u / \partial x^{2}$ аналогичное свойство справедливо в метрике пространства $\mathrm{L}_{2}(0,1)$. Теорема 1.1 полностью доказана.

Проделанный анализ показывает, что на многообразии $\Sigma$ в достаточно малой окрестности нуля определен локальный поток

$$
\Pi^{t}\left(u_{0}, u_{1}\right)=\left(u(t, x), \frac{\partial u}{\partial t}(t, x)\right),
$$

где $u$ - решение задачи Коши (1.3), (1.4), (1.10). Далее, опираясь на развитые при обосновании теоремы 1.1 конструкции, нетрудно убедиться, что поток (1.26) имеет любую конечную гладкость как по параметрам $\varepsilon, \varkappa, K$, так и по начальному условию $\left(u_{0}, u_{1}\right) \in \Sigma$. А это, в свою очередь, позволяет распространить на краевую задачу (1.3), (1.4) без каких-либо существенных изменений основные положения качественной теории обыкновенных дифференциальных уравнений: теоремы Ляпунова и Андронова-Витта об устойчивости по первому приближению, результаты об интегральных многообразиях и т.д.

1.3. Линейный анализ. Основным объектом исследования в настоящей работе является краевая задача

$$
\begin{gathered}
\frac{\partial^{2}}{\partial t^{2}}\left(u-\varkappa \frac{\partial^{2} u}{\partial x^{2}}\right)+\varepsilon \frac{\partial}{\partial t}\left(u-\varkappa \frac{\partial^{2} u}{\partial x^{2}}\right)=\frac{\partial^{2} u}{\partial x^{2}} \\
\left.\frac{\partial u}{\partial x}\right|_{x=1}=0,\left.\quad u\right|_{x=0}+\left.\left(1+\varepsilon^{2} \gamma\right) u\right|_{x=1}-\left.u^{3}\right|_{x=1}=0
\end{gathered}
$$


при условии, что параметр $\varepsilon>0$, характеризующий активные потери в линии, мал, а остальные параметры $\varkappa, \gamma>0$ имеют порядок единицы. Точнее говоря, будем интересоваться вопросами существования и устойчивости ее циклов и торов, бифурцирующих из нулевого состояния равновесия при увеличении $\gamma$. Однако для того чтобы убедиться в осмысленности данной постановки проблемы, сначала необходимо разобраться со свойствами устойчивости нулевого решения этой краевой задачи.

Итак, отбросим во втором граничном условии из (1.27) нелинейное слагаемое и положим в получившейся линейной краевой задаче $u=h(x) \exp \lambda t$. В результате приходим к задаче для $h(x)$

$$
h^{\prime \prime}-\mu^{2} h=0, \quad h^{\prime}(1)=0, \quad h(0)+\left(1+\varepsilon^{2} \gamma\right) h(1)=0,
$$

где $\mu^{2}=\left(\lambda^{2}+\varepsilon \lambda\right) /\left(1+\varepsilon \varkappa \lambda+\varkappa \lambda^{2}\right)$. Заметим далее, что решение уравнения из (1.28), удовлетворяющее первому граничному условию, имеет вид $h=$ $c \operatorname{ch}[\mu(x-1)]$, где $c \neq 0$ - произвольная постоянная. Подставляя его во второе граничное условие, для определения спектра устойчивости нулевого состояния равновесия имеем характеристическое уравнение

$$
\operatorname{ch} \mu+1+\gamma \varepsilon^{2}=0 .
$$

Будем считать сначала $\mu$ в уравнении (1.29) независимой комплексной переменной. Несложный анализ показывает, что это уравнение имеет два семейства корней

$$
\begin{gathered}
\pm \mu_{n}(\varepsilon), \quad \pm \bar{\mu}_{n}(\varepsilon), \quad n=1,2, \ldots, \\
\mu_{n}=i \omega_{n}+\mu_{*}(\varepsilon), \quad \mu_{*}(\varepsilon)=\sqrt{2 \gamma} \varepsilon+O\left(\varepsilon^{2}\right),
\end{gathered}
$$

где числа $\omega_{n}, n \geqslant 1$, те же, что и в (1.6)-(1.8), а $\mu_{*}$ - положительный корень уравнения $\operatorname{ch} \mu=1+\varepsilon^{2} \gamma$. Вспоминая затем о связи между $\lambda$ и $\mu$, убеждаемся, что собственные значения $\lambda$ краевой задачи (1.28) определяются из последовательности квадратных уравнений

$$
\left(1+\varepsilon \varkappa \lambda+\varkappa \lambda^{2}\right) \mu_{n}^{2}(\varepsilon)=\lambda^{2}+\varepsilon \lambda, \quad n \geqslant 1,
$$

а также уравнений, получающихся из (1.30) при замене $\mu_{n}$ на $\bar{\mu}_{n}$. И наконец, решая уравнения (1.30), приходим к выводу, что их корни $\lambda_{n}^{1}(\varepsilon), \lambda_{n}^{2}(\varepsilon)$ допускают равномерную по $n \geqslant 1$ асимптотику

$$
\begin{aligned}
& \lambda_{n}^{1}=i \sigma_{n}+\left(\frac{\sqrt{2 \gamma}}{\left(1+\varkappa \omega_{n}^{2}\right)^{3 / 2}}-\frac{1}{2}\right) \varepsilon+O\left(\varepsilon^{2}\right), \\
& \lambda_{n}^{2}=-i \sigma_{n}-\left(\frac{\sqrt{2 \gamma}}{\left(1+\varkappa \omega_{n}^{2}\right)^{3 / 2}}+\frac{1}{2}\right) \varepsilon+O\left(\varepsilon^{2}\right),
\end{aligned}
$$

где $\sigma_{n}=\omega_{n} / \sqrt{1+\varkappa \omega_{n}^{2}}$, а при $n \rightarrow \infty$ стремятся к конечным пределам

$$
-\frac{\varepsilon}{2} \pm i \sqrt{\frac{1}{\varkappa}-\frac{\varepsilon^{2}}{4}} .
$$


Из формул (1.31), (1.32) следует, что $\operatorname{Re} \lambda_{n}^{2} \leqslant-\varepsilon / 2, n \geqslant 1$. Что же касается корней $\lambda_{n}^{1}, n \geqslant 1$, то при увеличении параметра $\gamma$ и при прохождении его через критические значения

$$
\gamma_{n}=\frac{1}{8}\left(1+\varkappa \omega_{n}^{2}\right)^{3}, \quad n \geqslant 1
$$

они последовательно переходят в правую комплексную полуплоскость $\{\lambda$ : $\operatorname{Re} \lambda>0\}$. Таким образом, становится понятной причина специального выбора коэффициента усиления $K$ в краевой задаче (1.27) и обретает смысл вопрос о существовании и устойчивости ее периодических по $t$ решений, бифурцирующих из нуля при прохождении $\gamma$ через значения (1.33). Решению этого вопроса посвящен следующий параграф.

\section{§ 2. Явление буферности и механизмы его возникновения}

2.1. Основной результат. Из проделанного выше анализа следует, что линейная краевая задача

$$
\Pi(u) \equiv \frac{\partial^{2}}{\partial t^{2}}\left(u-\varkappa \frac{\partial^{2} u}{\partial x^{2}}\right)-\frac{\partial^{2} u}{\partial x^{2}}=0,\left.\quad \frac{\partial u}{\partial x}\right|_{x=1}=0,\left.\quad u\right|_{x=0}+\left.u\right|_{x=1}=0,
$$

получающаяся из (1.27) при $\varepsilon=0$ и при отбрасывании кубического слагаемого во втором граничном условии, допускает семейство периодических решений

$$
u=\exp \left( \pm i \sigma_{n} t\right) \cos \omega_{n} x, \quad n \geqslant 1
$$

где частоты $\sigma_{n}$ определены в (1.31). Обыгрывая этот факт, зафиксируем произвольно натуральное $n$ и будем строить асимптотику цикла краевой задачи $(1.27)$ с частотой, близкой к $\sigma_{n}$, в виде рядов по целым степеням $\varepsilon$ :

$$
u=\varepsilon u_{0}(\tau, x)+\varepsilon^{2} u_{1}(\tau, x)+\varepsilon^{3} u_{2}(\tau, x)+\cdots, \quad \tau=\left(1+\varepsilon \delta_{1}+\varepsilon^{2} \delta_{2}+\cdots\right) t,
$$

где

$$
u_{0}=\xi\left[\exp \left(i \sigma_{n} \tau\right)+\exp \left(-i \sigma_{n} \tau\right)\right] \cos \omega_{n} x,
$$

все функции $u_{j}, j \geqslant 1$, периодичны по $\tau$ с периодом $2 \pi / \sigma_{n}$, а $\xi, \delta_{1}, \delta_{2}$ и т.д. подлежащие определению вещественные постоянные.

Подставляя соотношения (2.3), (2.4) в задачу (1.27) и приравнивая последовательно коэффициенты при одинаковых степенях $\varepsilon$, для нахождения $u_{j}, j \geqslant 1$, получаем серию линейных неоднородных краевых задач вида

$$
\Pi\left(u_{j}\right)=f_{j}(\tau, x),\left.\quad \frac{\partial u_{j}}{\partial x}\right|_{x=1}=0,\left.\quad u_{j}\right|_{x=0}+\left.u_{j}\right|_{x=1}=\psi_{j}(\tau),
$$

где П - дифференциальный оператор из (2.1), в котором $t$ заменено на $\tau$. Что же касается решений $u_{j}$ этих краевых задач, то их будем искать в том же виде, что и соответствующие неоднородности, т.е. в классе нечетных тригонометрических полиномов переменной $\sigma_{n} \tau$ степени не выше $2 j-1$. 
На первом шаге алгоритма, т.е. при $j=1$, рассмотрению подлежит краевая задача (2.5) с неоднородностями

$$
f_{1}=-2 \delta_{1} \frac{\partial^{2}}{\partial \tau^{2}} \mathscr{L} u_{0}-\frac{\partial}{\partial \tau} \mathscr{L} u_{0}, \quad \psi_{1}=0
$$

где $\mathscr{L} u=u-\varkappa \partial^{2} u / \partial x^{2}$. Несложный ее анализ приводит к выводу, что

$$
u_{1}=A_{1,1}(x) \exp \left(i \sigma_{n} \tau\right)+\bar{A}_{1,1}(x) \exp \left(-i \sigma_{n} \tau\right)
$$

где $A_{1,1}(x)$ - решение краевой задачи

$$
\begin{aligned}
A_{1,1}^{\prime \prime}+\omega_{n}^{2} A_{1,1} & =\left(1+\varkappa \omega_{n}^{2}\right)^{2}\left(-2 \delta_{1} \sigma_{n}^{2}+i \sigma_{n}\right) \xi \cos \omega_{n} x, \\
A_{1,1}^{\prime}(1) & =0, \quad A_{1,1}(0)+A_{1,1}(1)=0 .
\end{aligned}
$$

Остается добавить, что в силу отмеченных выше свойств оператора (1.5) задача (2.7) оказывается заведомо разрешимой, а ее решение задается равенствами

$$
\begin{gathered}
A_{1,1}(x)=\frac{\left(1+\varkappa \omega_{n}^{2}\right)^{2}}{\omega_{n}}\left(\sigma_{n}+2 i \delta_{1} \sigma_{n}^{2}\right) \xi B_{n}(x)+\eta_{1} \cos \omega_{n} x, \\
B_{n}(x)=\frac{i}{2}(x-1) \sin \omega_{n} x
\end{gathered}
$$

где $\eta_{1} \in \mathbb{R}$ - произвольная постоянная.

При $j=2$ имеем дело с краевой задачей $(2.5)$, в которой

$$
\begin{gathered}
f_{2}=-\left(\delta_{1}^{2}+2 \delta_{2}\right) \frac{\partial^{2}}{\partial \tau^{2}} \mathscr{L} u_{0}-2 \delta_{1} \frac{\partial^{2}}{\partial \tau^{2}} \mathscr{L} u_{1}-\frac{\partial}{\partial \tau} \mathscr{L} u_{1}-\delta_{1} \frac{\partial}{\partial \tau} \mathscr{L} u_{0}, \\
\psi_{2}=\left.\left(-\gamma u_{0}+u_{0}^{3}\right)\right|_{x=1} .
\end{gathered}
$$

А отсюда и из $(2.4),(2.6)$ заключаем, что ее решение $u_{2}$ следует искать в виде

$$
\begin{aligned}
u_{2}= & A_{2,1}(x) \exp \left(i \sigma_{n} \tau\right)+\bar{A}_{2,1}(x) \exp \left(-i \sigma_{n} \tau\right) \\
& +A_{2,3}(x) \exp \left(3 i \sigma_{n} \tau\right)+\bar{A}_{2,3}(x) \exp \left(-3 i \sigma_{n} \tau\right)
\end{aligned}
$$

Таким образом, проблема сводится к определению фигурирующих в (2.11) коэффициентов $A_{2,1}, A_{2,3}$ из аналогичных (2.7) линейных неоднородных краевых задач.

Анализ формул (2.10) приводит к равенству

$$
A_{2,1}(x)=\frac{\left(1+\varkappa \omega_{n}^{2}\right)^{2}}{\omega_{n}}\left(\sigma_{n} \delta_{1}+i\left(\delta_{1}^{2}+2 \delta_{2}\right) \sigma_{n}^{2}\right) \xi B_{n}(x)+\eta_{2} \cos \omega_{n} x+\widetilde{A}_{2,1}(x),
$$

в котором $B_{n}$ - функция $(2.9), \eta_{2} \in \mathbb{R}$ - произвольная постоянная, а функция $\widetilde{A}_{2,1}$ - решение краевой задачи

$$
\begin{gathered}
\widetilde{A}_{2,1}^{\prime \prime}+\omega_{n}^{2} \widetilde{A}_{2,1}=\widetilde{f}(x), \quad \widetilde{A}_{2,1}^{\prime}(1)=0, \quad \widetilde{A}_{2,1}(0)+\widetilde{A}_{2,1}(1)=\widetilde{\psi}, \\
\tilde{f}=\left(i \sigma_{n}-2 \delta_{1} \sigma_{n}^{2}\right)\left(1+\varkappa \omega_{n}^{2}\right)\left(A_{1,1}-\varkappa A_{1,1}^{\prime \prime}\right), \quad \widetilde{\psi}=\gamma \xi-3 \xi^{3} .
\end{gathered}
$$


Заметим, однако, что в отличие от (2.7) получившаяся задача (2.13) не является разрешимой автоматически. Выполнения же условия ее разрешимости

$$
\int_{0}^{1} \tilde{f}(x) \sin \omega_{n} x d x=\omega_{n} \tilde{\psi}
$$

будем добиваться за счет подходящего выбора имеющихся в запасе произвольных постоянных $\delta_{1}, \xi$.

Подставляя в (2.15) формулы $(2.14)$, для $\xi, \delta_{1}$ получаем уравнение

$$
\left(\gamma-\frac{1}{8} \frac{\left(1+\varkappa \omega_{n}^{2}\right)^{4}}{\omega_{n}^{2}}\left(\sigma_{n}+2 i \delta_{1} \sigma_{n}^{2}\right)^{2}\right) \xi-3 \xi^{3}=0 .
$$

Предположим, далее, что выполнено условие самовозбуждения автоколебаний на частоте $\sigma_{n}$, т.е. неравенство

$$
\gamma>\gamma_{n}
$$

где $\gamma_{n}$ - критическое значение (1.33). Тогда из (2.16) находим

$$
\delta_{1}=0, \quad \xi=\sqrt{\frac{\gamma-\gamma_{n}}{3}},
$$

а затем, решая задачу (2.13), определяем фигурирующую в (2.12) функцию $\widetilde{A}_{2,1}(x)$. Однако поскольку в дальнейшем явный вид $\widetilde{A}_{2,1}$ нам не потребуется, то соответствующие вычисления опустим.

Для коэффициента $A_{2,3}$ из (2.11) получается краевая задача

$$
\left(1-8 \varkappa \omega_{n}^{2}\right) A_{2,3}^{\prime \prime}+9 \omega_{n}^{2} A_{2,3}=0, \quad A_{2,3}^{\prime}(1)=0, \quad A_{2,3}(0)+A_{2,3}(1)=-\xi^{3},
$$

которая при некоторой общности положения оказывается невырожденной. Действительно, пусть при всех $m \geqslant 3 n$ выполняются условия

$$
\varkappa \neq \frac{1}{8 \omega_{n}^{2}}-\frac{9}{8 \omega_{m}^{2}}, \quad \varkappa \neq \frac{1}{8 \omega_{n}^{2}},
$$

означающие отсутствие резонансов вида $3 \sigma_{n}=\sigma_{m}, m \geqslant 3 n$. Тогда из (2.19) имеем

$$
A_{2,3}=c \cos \frac{3 \omega_{n}(x-1)}{\sqrt{1-8 \varkappa \omega_{n}^{2}}}, \quad c=-\xi^{3}\left(1+\cos \frac{3 \omega_{n}}{\sqrt{1-8 \varkappa \omega_{n}^{2}}}\right)^{-1} .
$$

Подведем некоторый итог. При выполнении условий (2.17), (2.20) удается построить первые три приближения $u_{j}, j=0,1,2$, причем $u_{0}$ определяется однозначно, а две другие функции - с точностью до слагаемых

$$
\eta_{j}\left[\exp \left(i \sigma_{n} \tau\right)+\exp \left(-i \sigma_{n} \tau\right)\right] \cos \omega_{n} x
$$

(см. формулы (2.8), (2.12)). Как будет показано ниже, в принципе этой информации достаточно для исследования свойств устойчивости и доказательства самого факта существования у краевой задачи (1.27) цикла с асимптотикой $(2.3),(2.4),(2.18)$. Однако при необходимости описанный алгоритм можно продолжить. Действительно, разрешимости краевой задачи для коэффициента 
функции $u_{j+2}, j \geqslant 1$, при гармонике $\exp \left(i \sigma_{n} \tau\right)$ добиваемся за счет очередной поправки к частоте $\delta_{j+1}$ и слагаемого вида $(2.22)$, с точностью до которого известна функция $u_{j}$. Характерным здесь является то обстоятельство, что в отличие от $(2.16)$ для $\eta_{j}, \delta_{j+1}, j \geqslant 1$, получаются уже линейные неоднородные уравнения, из которых эти постоянные определяются однозначно. Что же касается коэффициентов функции $u_{j+2}$ при остальных гармониках, то, как и выше, при некоторой общности положения относительно параметра $\varkappa$ для них получаются невырожденные краевые задачи.

Доказательство существования у краевой задачи (1.27) цикла с построенной асимптотикой может быть проведено двумя способами. Один из подходов, носящий геометрический характер и базирующийся на известном принципе кольца [10], для широкого класса нелинейных волновых уравнений реализован в монографии [9]. Другой же способ, предложенный в [11] и являющийся чисто аналитическим, сводит интересующую нас проблему к нахождению неподвижной точки некоторого нелинейного интегрального уравнения в подходящем пространстве периодических функций. Однако применение любого из указанных методов требует предварительного анализа свойств устойчивости линейной краевой задачи

$$
\begin{gathered}
\frac{\partial^{2}}{\partial \tau^{2}}\left(u-\varkappa \frac{\partial^{2} u}{\partial x^{2}}\right)+\varepsilon \frac{\partial}{\partial \tau}\left(u-\varkappa \frac{\partial^{2} u}{\partial x^{2}}\right)=\frac{\partial^{2} u}{\partial x^{2}} \\
\left.\frac{\partial u}{\partial x}\right|_{x=1}=0,\left.\quad u\right|_{x=0}+\left.u\right|_{x=1}+\left.\varepsilon^{2}\left(\gamma-3 u_{0}^{2}(\tau, 1)\right) u\right|_{x=1}=0
\end{gathered}
$$

где $u_{0}$ - функция (2.4), получающейся из (1.27) при линеаризации на построенном выше приближенном цикле и отбрасывании несущественных для дальнейшего слагаемых порядка $\varepsilon^{3}$. Последняя же проблема решается с помощью специальных алгоритмов, описание которых приводится ниже.

Начнем с расчета характеристических показателей краевой задачи (2.23) на собственной частоте $\sigma_{n}$ приближенного периодического решения (2.3), (2.4). С этой целью подставим в (2.23) соотношение

$$
u=\left[w_{0, n}(\tau, x)+\varepsilon w_{1, n}(\tau, x)+\varepsilon^{2} w_{2, n}(\tau, x)\right] \exp (\varepsilon \lambda \tau),
$$

где $w_{j, n}, j=0,1,2,-$ тригонометрические полиномы переменной $\sigma_{n} \tau$,

$$
w_{0, n}=\left[a_{1} \exp \left(i \sigma_{n} \tau\right)+a_{2} \exp \left(-i \sigma_{n} \tau\right)\right] \cos \omega_{n} x
$$

а $\lambda, a_{1}, a_{2}$ - подлежащие определению комплексные постоянные. Приравнивая затем коэффициенты при $\varepsilon$ и $\varepsilon^{2}$, для нахождения $w_{1, n}$ и $w_{2, n}$ получаем краевые задачи вида (2.5), первая из которых разрешима в нужном классе функций. Поэтому для $w_{1, n}$ приведем сразу окончательный результат:

$$
\begin{gathered}
w_{1, n}=C_{1, n, 1}^{+}(x) \exp \left(i \sigma_{n} \tau\right)+C_{1, n, 1}^{-}(x) \exp \left(-i \sigma_{n} \tau\right), \\
C_{1, n, 1}^{+}=a_{1} W_{0}(x), \quad C_{1, n, 1}^{-}=-a_{2} W_{0}(x), \\
W_{0}(x)=\frac{\left(1+\varkappa \omega_{n}^{2}\right)^{2}}{\omega_{n}} \sigma_{n}(2 \lambda+1) B_{n}(x),
\end{gathered}
$$

где $B_{n}-$ функция $(2.9)$. 
В случае $w_{2, n}$ анализу подлежит краевая задача

$$
\begin{gathered}
\Pi\left(w_{2, n}\right)+(2 \lambda+1) \frac{\partial}{\partial \tau} \mathscr{L} w_{1, n}+\lambda(\lambda+1) \mathscr{L} w_{0, n}=0, \\
\left.\frac{\partial w_{2, n}}{\partial x}\right|_{x=1}=0,\left.\quad w_{2, n}\right|_{x=0}+\left.w_{2, n}\right|_{x=1}+\left.\left(\gamma-3 u_{0}^{2}(\tau, 1)\right) w_{0, n}\right|_{x=1}=0 .
\end{gathered}
$$

Учитывая в неоднородностях из (2.27), (2.28) явные формулы для $w_{0, n}, w_{1, n}$ (см. (2.25), (2.26)), приходим к выводу, что решение данной задачи следует искать в виде

$$
\begin{aligned}
w_{2, n}=C_{2, n, 1}^{+}(x) \exp \left(i \sigma_{n} \tau\right)+C_{2, n, 1}^{-}(x) \exp \left(-i \sigma_{n} \tau\right) & \\
& +C_{2, n, 3}^{+}(x) \exp \left(3 i \sigma_{n} \tau\right)+C_{2, n, 3}^{-}(x) \exp \left(-3 i \sigma_{n} \tau\right)
\end{aligned}
$$

В итоге для коэффициентов $C_{2, n, 1}^{ \pm}$из (2.29) имеем аналогичные (2.13) вырожденные краевые задачи, условия разрешимости которых задаются равенствами

$$
\left(\gamma-6 \xi^{2}\right) a_{1}-3 \xi^{2} a_{2}=\gamma_{n}(2 \lambda+1)^{2} a_{1}, \quad-3 \xi^{2} a_{1}+\left(\gamma-6 \xi^{2}\right) a_{2}=\gamma_{n}(2 \lambda+1)^{2} a_{2},
$$

где, напомним, $\xi$ - амплитуда из (2.18), $\gamma_{n}$ - критическое значение (1.33). Что же касается коэффициентов $C_{2, n, 3}^{ \pm}$, то они определяются из аналогичных $(2.19)$ краевых задач, которые в силу условий (2.20) оказываются невырожденными.

Будем рассматривать равенства (2.30) как систему для нахождения коэффициентов $a_{1}, a_{2}$. Очевидно, она допускает нетривиальные решения в том и только в том случае, когда $\lambda$ является корнем одного из уравнений

$$
(2 \lambda+1)^{2}=1, \quad(2 \lambda+1)^{2}=\frac{\gamma-9 \xi^{2}}{\gamma_{n}} .
$$

А отсюда, в свою очередь, заключаем, что система (2.30) имеет четыре набора решений

$$
\begin{aligned}
& \lambda=\lambda_{1}, \quad a_{1}=i, \quad a_{2}=-i ; \quad \lambda=\lambda_{2}, \quad a_{1}=i, \quad a_{2}=-i ; \\
& \lambda=\lambda_{3}, \quad a_{1}=a_{2}=1 ; \quad \lambda=\lambda_{4}, \quad a_{1}=a_{2}=1,
\end{aligned}
$$

где

$$
\begin{aligned}
\lambda_{1}=0, & \lambda_{2}=-1, \\
\lambda_{3}=\frac{-1+\sqrt{\left(\gamma-9 \xi^{2}\right) / \gamma_{n}}}{2}, & \lambda_{4}=-\frac{1+\sqrt{\left(\gamma-9 \xi^{2}\right) / \gamma_{n}}}{2} .
\end{aligned}
$$

Отметим еще, что наличие среди корней уравнений (2.31) нуля вполне естественно, так как (2.23) - линеаризация на приближенном цикле. Все же остальные корни (2.33) находятся в полуплоскости $\{\lambda: \operatorname{Re} \lambda<0\}$.

При расчете характеристических показателей задачи (2.23) на частотах $\sigma_{m}$, $m \neq n, m \geqslant 1$, подставим в нее аналогичное (2.24) соотношение

$$
u=\left[\cos \omega_{m} x+\varepsilon w_{1, m}+\varepsilon^{2} w_{2, m}\right] \exp \left[\left(i \sigma_{m}+\varepsilon \lambda_{m, 1}+\varepsilon^{2} \lambda_{m, 2}\right) \tau\right],
$$

где, как и выше, функции $w_{j, m}, j=1,2$, являются тригонометрическими полиномами переменной $\sigma_{n} \tau$, а постоянные $\lambda_{m, 1}, \lambda_{m, 2}$ подлежат определению. 
В результате, приравнивая коэффициенты при $\varepsilon$, для $w_{1, m}$ приходим к задаче

$$
\begin{gathered}
\left(\frac{\partial^{2}}{\partial \tau^{2}}+2 i \sigma_{m} \frac{\partial}{\partial \tau}-\sigma_{m}^{2}\right)\left(w_{1, m}-\varkappa \frac{\partial^{2} w_{1, m}}{\partial x^{2}}\right)-\frac{\partial^{2} w_{1, m}}{\partial x^{2}} \\
=-i \sigma_{m}\left(2 \lambda_{m, 1}+1\right)\left(1+\varkappa \omega_{m}^{2}\right) \cos \omega_{m} x \\
\left.\frac{\partial w_{1, m}}{\partial x}\right|_{x=1}=0,\left.\quad w_{1, m}\right|_{x=0}+\left.w_{1, m}\right|_{x=1}=0 .
\end{gathered}
$$

Несложный ее анализ приводит к равенству

$$
w_{1, m}=\sigma_{m}\left(2 \lambda_{m, 1}+1\right) \frac{\left(1+\varkappa \omega_{m}^{2}\right)^{2}}{\omega_{m}} B_{m}(x),
$$

где $B_{m}$ - функция, получающаяся из (2.9) при замене $n$ на $m$.

При нахождении $w_{2, m}$ имеем дело с краевой задачей

$$
\begin{aligned}
& \Pi_{m}\left(w_{2, m}\right)+i \sigma_{m}\left(2 \lambda_{m, 1}+1\right)\left(w_{1, m}-\varkappa \frac{\partial^{2} w_{1, m}}{\partial x^{2}}\right) \\
& \quad+\left[\lambda_{m, 1}\left(\lambda_{m, 1}+1\right)+2 i \sigma_{m} \lambda_{m, 2}\right]\left(1+\varkappa \omega_{m}^{2}\right) \cos \omega_{m} x=0, \\
& \left.\frac{\partial w_{2, m}}{\partial x}\right|_{x=1}=0,\left.\quad w_{2, m}\right|_{x=0}+\left.w_{2, m}\right|_{x=1}=\gamma-3 u_{0}^{2}(\tau, 1),
\end{aligned}
$$

где $\Pi_{m}$ - дифференциальный оператор, порожденный левой частью уравнения из (2.35). Ее решение будем искать в виде тригонометрического многочлена той же структуры, что и соответствующие неоднородности, а значит (см. (2.4), (2.36)), в виде

$$
w_{2, m}=C_{2, m, 0}(x)+C_{2, m, 2}^{+}(x) \exp \left(2 i \sigma_{n} \tau\right)+C_{2, m, 2}^{-}(x) \exp \left(-2 i \sigma_{n} \tau\right) .
$$

Остановимся на способе определения фигурирующих в (2.38) коэффициентов $C_{2, m, 0}, C_{2, m, 2}^{ \pm}$. Анализируя структуру входящих в $(2.37)$ неоднородностей, для $C_{2, m, 0}$ получаем краевую задачу

$$
C_{2, m, 0}^{\prime \prime}+\omega_{m}^{2} C_{2, m, 0}=f_{m}(x), \quad C_{2, m, 0}^{\prime}(1)=0, \quad C_{2, m, 0}(0)+C_{2, m, 0}(1)=\psi_{m},
$$

где

$$
\begin{gathered}
f_{m}(x)=\left(1+\varkappa \omega_{m}^{2}\right)^{2}\left\{i \sigma_{m}^{2}\left(2 \lambda_{m, 1}+1\right)^{2} \frac{\left(1+\varkappa \omega_{m}^{2}\right)^{2}}{\omega_{m}} B_{m}(x)\right. \\
\left.+\left[\lambda_{m, 1}\left(\lambda_{m, 1}+1\right)+\varkappa \omega_{m}^{2}\left(2 \lambda_{m, 1}+1\right)^{2}+2 i \sigma_{m} \lambda_{m, 2}\right] \cos \omega_{m} x\right\} \\
\psi_{m}=\gamma-6 \xi^{2}
\end{gathered}
$$

Заметим, далее, что условие разрешимости задачи (2.39) задается аналогичным (2.15) равенством, которое после подстановки в него формул (2.40) преобразуется к виду

$$
\gamma_{m}\left(2 \lambda_{m, 1}+1\right)^{2}=\gamma-6 \xi^{2}
$$

Получившееся равенство будем рассматривать как уравнение для нахождения фигурирующего в (2.34) свободного параметра $\lambda_{m, 1}$. Нетрудно увидеть, 
что из (2.41) определяются два возможных значения $\lambda_{m, 1}^{(1)}, \lambda_{m, 1}^{(2)}$ этого параметра, причем при выполнении условия

$$
\gamma>2 \gamma_{n}-\gamma_{m}
$$

имеем $\operatorname{Re} \lambda_{m, 1}^{(k)}<0, k=1,2$. В случае же выполнения строго противоположного (2.42) условия корни уравнения (2.41) вещественны и $\lambda_{m, 1}^{(1)} \lambda_{m, 1}^{(2)}<0$.

После нахождения $\lambda_{m, 1}$ распорядимся имеющейся в запасе постоянной $\lambda_{m, 2}$ таким образом, чтобы занулился коэффициент перед $\cos \omega_{m} x$ в неоднородности $f_{m}(x)$ из (2.40) (осмысленность такого выбора $\lambda_{m, 2}$ прояснится в дальнейшем). В итоге приходим к равенствам

$$
\begin{gathered}
\lambda_{m, 2}=\frac{i}{2 \sigma_{m}}\left[\lambda_{m, 1}\left(\lambda_{m, 1}+1\right)+\varkappa \omega_{m}^{2}\left(2 \lambda_{m, 1}+1\right)^{2}\right], \\
C_{2, m, 0}(x)=\left(\gamma-6 \xi^{2}\right)\left[(x-1)^{2} \cos \omega_{m} x-\frac{1}{\omega_{m}}(x-1) \sin \omega_{m} x\right] .
\end{gathered}
$$

Оставшиеся коэффициенты $C_{2, m, 2}^{ \pm}(x)$ из $(2.38)$ при некоторой общности положения, связанной с параметром $\varkappa$, определяются однозначно из соответствующих невырожденных краевых задач. Поэтому в данном случае приведем сразу итоговые формулы:

$$
\begin{gathered}
C_{2, m, 2}^{ \pm}(x)=r_{m}^{ \pm} \cos \left[\beta_{m}^{ \pm}(x-1)\right], \quad r_{m}^{ \pm}=-\frac{3 \xi^{2}}{1+\cos \beta_{m}^{ \pm}}, \\
\beta_{m}^{ \pm}=\frac{\sigma_{m} \pm 2 \sigma_{n}}{\sqrt{1-\varkappa\left(\sigma_{m} \pm 2 \sigma_{n}\right)^{2}}} .
\end{gathered}
$$

Кроме того, предполагаем, что при любых целых $m \geqslant 1, m \neq n$ и $k \geqslant 1$ выполняются неравенства

$$
\left(\sigma_{m} \pm 2 \sigma_{n}\right)^{2}-\sigma_{k}^{2} \neq 0, \quad\left(\sigma_{m} \pm 2 \sigma_{n}\right)^{2}-\frac{1}{\varkappa} \neq 0, \quad\left(\frac{1}{\sqrt{\varkappa}} \pm 2 \sigma_{n}\right)^{2}-\sigma_{k}^{2} \neq 0,
$$

где, напомним, номер $n$ фиксирован. Эти неравенства, как нетрудно увидеть, обеспечивают отличие от нуля знаменателей в (2.44) и их стремление к ненулевым конечным пределам при $m \rightarrow \infty$.

Для того чтобы сформулировать основной результат настоящей статьи, введем в рассмотрение числа

$$
R_{n, m}=2 \gamma_{n}-\gamma_{m}-\gamma, \quad m \geqslant 1,
$$

знаки которых, как уже отмечалось выше, отвечают за расположение корней уравнений (2.41). Справедливо следующее утверждение.

ТЕОРема 2.1. Пусть при некотором натуральном $n$ выполняются неравенства (2.17), (2.20), (2.45), а также условия $\gamma \neq 2 \gamma_{n}, 3 \gamma_{n} / 2$. Пусть, далее, отличны от нуля числа (2.46) и количество положительных среди них равно $m_{0}$. Тогда найдется такое достаточно малое $\varepsilon_{0}>0$, что при всех $0<$ $\varepsilon \leqslant \varepsilon_{0}$ краевая задача (1.27) имеет иикл с асимптотикой (2.3), (2.4), (2.18), экспоненииально орбитально устойчивый при $m_{0}=0$ и дихотомичный в случае $m_{0}>0$ с размерностью неустойчивого многообразия $2 m_{0}+1$. 
Сформулированная теорема позволяет выявить два различных механизма возникновения буферности в краевой задаче (1.27). Первый механизм реализуется при фиксированном $\varkappa$ и при увеличении параметра $\gamma$. Действительно, цикл $(2.3),(2.4)$ с номером $n$ бифурцирует из нуля при $\gamma>\gamma_{n}$, а затем, подрастая по амплитуде, становится устойчивым при

$$
\gamma>2 \gamma_{n}-\gamma_{1}
$$

Таким образом, при надлежащем увеличении $\gamma$, уменьшении $\varepsilon$ и при некоторой общности положения, связанной с параметром $\varkappa$, можно добиться сосуществования у рассматриваемой задачи любого наперед заданного конечного числа устойчивых циклов, т.е. наблюдается феномен буферности.

Второй механизм связан с уменьшением $\varkappa$ при фиксированном $\gamma>1 / 8$. В этом случае в силу очевидных предельных равенств $\lim _{\varkappa \rightarrow 0} \gamma_{n}=1 / 8$ условия устойчивости (2.47) заведомо выполняются при подходящем уменьшении $\varkappa$ для номеров $n=1,2, \ldots, N$, где $N$ произвольно фиксировано.

2.2. Доказательство теоремы 2.1. Как показано в монографии [9], центральным моментом доказательства утверждений, подобных теореме 2.1, является обоснование алгоритма исследования устойчивости соответствующей системы в вариациях на приближенном цикле. Точнее говоря, в ряде случаев с помощью найденных в ходе алгоритма приближенных решений Ляпунова-Флоке удается сконструировать корректную бесконечномерную замену переменных, приводящую исходную систему к некоторой линейной системе в пространстве последовательностей $l_{2}$ с близкими к постоянным периодическими коэффициентами. В настоящем пункте подобного рода замена переменных строится для краевой задачи (2.23).

Прежде всего введем в рассмотрение фазовое пространство задачи (2.23). Таковым является $\left(2 \pi / \sigma_{n}\right)$-периодическое по $\tau$ линейное подпространство

$$
\Sigma(\tau, \varepsilon) \subset \mathrm{W}_{2}^{2}(0,1) \times \mathrm{W}_{2}^{2}(0,1),
$$

состоящее из пар функций $\left(h_{1}(x), h_{2}(x)\right)$, удовлетворяющих граничным условиям

$$
\begin{gathered}
h_{1}^{\prime}(1)=h_{2}^{\prime}(1)=0,\left.\quad h_{1}\right|_{x=0}+\left.h_{1}\right|_{x=1}+\left.\varepsilon^{2}\left(\gamma-3 u_{0}^{2}(\tau, 1)\right) h_{1}\right|_{x=1}=0, \\
\left.h_{2}\right|_{x=0}+\left.h_{2}\right|_{x=1}+\left.\varepsilon^{2}\left(\gamma-3 u_{0}^{2}(\tau, 1)\right) h_{2}\right|_{x=1}-\left.6 \varepsilon^{2} u_{0}(\tau, 1) \frac{\partial u_{0}}{\partial \tau}(\tau, 1) h_{1}\right|_{x=1}=0 .
\end{gathered}
$$

Термин “фазовое пространство” в данном случае имеет следующий смысл. Опираясь на развитые в $\S 1$ конструкции, можно показать, что если дополнить краевую задачу (2.23) произвольными начальными условиями

$$
\left.u\right|_{\tau=\tau_{0}}=h_{1}(x),\left.\quad \frac{\partial u}{\partial \tau}\right|_{\tau=\tau_{0}}=h_{2}(x), \quad\left(h_{1}, h_{2}\right) \in \Sigma\left(\tau_{0}, \varepsilon\right),
$$

то для соответствующего ее решения $u=u(\tau, x)$ при всех $\tau \in \mathbb{R}$ справедливо включение

$$
\left(u(\tau, x), \frac{\partial u}{\partial \tau}(\tau, x)\right) \in \Sigma(\tau, \varepsilon) .
$$


Первый этап обоснования изложенного в п. 2.1 алгоритма исследования устойчивости состоит в построении $\left(2 \pi / \sigma_{n}\right)$-периодического базиса в $\Sigma(\tau, \varepsilon)$. С этой целью рассмотрим фигурирующие в (2.34) функции

$$
\cos \omega_{m} x+\varepsilon w_{1, m}(x)+\varepsilon^{2} w_{2, m}(\tau, x), \quad m \geqslant 1, \quad m \neq n .
$$

Из способа построения $w_{1, m}, w_{2, m}$ заключаем, что при подстановке выражений (2.48) в левую часть второго граничного условия из (2.23) получаются "невязки" вида $\varepsilon^{3} \widetilde{w}_{m}(\tau, \varepsilon)$, где $\widetilde{w}_{m}$ - тригонометрические полиномы переменной $\sigma_{n} \tau$ четвертой степени с равномерно ограниченными по $\varepsilon, m$ коэффициентами.

Добавим к сумме (2.48) еще одно слагаемое

$$
-\frac{\varepsilon^{3} \widetilde{w}_{m}(\tau, \varepsilon) \cos \left(\left(\omega_{m}+\omega_{1}\right) x\right)}{2+\varepsilon^{2}\left(\gamma-3 u_{0}^{2}(\tau, 1)\right)}
$$

и результат при $\lambda_{m, 1}=\lambda_{m, 1}^{(1)}$ и $\lambda_{m, 1}=\lambda_{m, 1}^{(2)}$, где, напомним, $\lambda_{m, 1}^{(j)}, j=1,2,-$ корни уравнения (2.41), обозначим соответственно через $w_{m}^{(1)}(\tau, x, \varepsilon), w_{m}^{(2)}(\tau, x, \varepsilon)$. Введем затем в рассмотрение вектор-функции

$$
e_{m}^{(j)}(\tau, x, \varepsilon), \quad \bar{e}_{m}^{(j)}(\tau, x, \varepsilon), \quad j=1,2, \quad m \geqslant 1, \quad m \neq n,
$$

где черта означает комплексное сопряжение,

$$
e_{m}^{(j)}=\frac{1}{\omega_{m}^{2}}\left(w_{m}^{(j)},\left(i \sigma_{m}+\varepsilon \lambda_{m, 1}^{(j)}+\varepsilon^{2} \lambda_{m, 2}\right) w_{m}^{(j)}+\frac{\partial w_{m}^{(j)}}{\partial \tau}\right) .
$$

Обращаем внимание, что в (2.51) у постоянной $\lambda_{m, 2}$ отсутствует верхний индекс $(j)$, так как в силу (2.43) она принимает одно и то же значение для обоих корней уравнения (2.41). Отметим также, что из способа подправления сумм (2.48) очевидным образом следует принадлежность функций (2.50) пространству $\Sigma(\tau, \varepsilon)$ (точнее говоря, его комплексификации) при всех $\tau \in \mathbb{R}$.

При $m=n$ рассмотрим суммы

$$
w_{0, n}(\tau, x)+\varepsilon w_{1, n}(\tau, x)+\varepsilon^{2} w_{2, n}(\tau, x)
$$

из (2.24), отвечающие четырем возможным вариантам выбора параметров $\lambda$, $a_{1}, a_{2}$ (см. (2.32)), и подправим их слагаемыми вида (2.49). В результате получим четыре функции, которые обозначим соответственно через $w_{n}^{(j)}(\tau, x, \varepsilon)$, $j=1,2,3,4$. После этого дополним семейство вектор-функций (2.50) еще четырьмя элементами из $\Sigma(\tau, \varepsilon)$ :

$$
e_{n}^{(j)}(\tau, x, \varepsilon)=\left(w_{n}^{(j)}, \varepsilon \lambda_{j} w_{n}^{(j)}+\frac{\partial w_{n}^{(j)}}{\partial \tau}\right), \quad j=1,2,3,4,
$$

где числа $\lambda_{j}$ определены в (2.32). 
Полученная система $(2.50),(2.52)$ и является искомым $\left(2 \pi / \sigma_{n}\right)$-периодическим базисом в $\Sigma(\tau, \varepsilon)$. Для доказательства этого факта перейдем к эквивалентной системе

$$
\begin{gathered}
g_{m}^{(1)}=e_{m}^{(1)}, \bar{g}_{m}^{(1)}, \quad g_{m}^{(2)}=\frac{e_{m}^{(1)}-e_{m}^{(2)}}{\varepsilon}, \bar{g}_{m}^{(2)}, \quad m=1,2, \ldots, \quad m \neq n \\
g_{n}^{(1)}=e_{n}^{(1)}, \quad g_{n}^{(2)}=\frac{e_{n}^{(1)}-e_{n}^{(2)}}{\varepsilon}, \quad g_{n}^{(3)}=e_{n}^{(3)}, \quad g_{n}^{(4)}=\frac{e_{n}^{(3)}-e_{n}^{(4)}}{\varepsilon} .
\end{gathered}
$$

Напомним, что две системы называются эквивалентными, если элементы одной из них выражаются через элементы другой и соответствующая бесконечномерная матрица перехода индуцирует линейный оператор в пространстве последовательностей $l_{2}$, ограниченный вместе со своим обратным. В нашем случае требуемая эквивалентность очевидна, так как матрица перехода между системами (2.52) и (2.53) имеет блочно-диагональную структуру.

Для выявления свойств системы (2.53) заметим, что при $\varepsilon=0$ она обращается в систему

$$
\begin{gathered}
\frac{1}{\omega_{m}^{2}}\left(1, i \sigma_{m}\right) \cos \omega_{m} x, \quad \frac{1}{\omega_{m}^{2}}\left(1,-i \sigma_{m}\right) \cos \omega_{m} x, \\
\frac{1}{\omega_{m}^{2}}\left(i \sigma_{m},-\sigma_{m}^{2}\right) r_{m}(x-1) \sin \omega_{m} x+\frac{1}{\omega_{m}^{2}}\left(0, \lambda_{m, 1}^{(1)}-\lambda_{m, 1}^{(2)}\right) \cos \omega_{m} x, \\
\frac{1}{\omega_{m}^{2}}\left(-i \sigma_{m},-\sigma_{m}^{2}\right) \bar{r}_{m}(x-1) \sin \omega_{m} x+\frac{1}{\omega_{m}^{2}}\left(0, \bar{\lambda}_{m, 1}^{(1)}-\bar{\lambda}_{m, 1}^{(2)}\right) \cos \omega_{m} x, \\
m \geqslant 1, \quad m \neq n ; \\
\left(-2 \sin \sigma_{n} \tau,-2 \sigma_{n} \cos \sigma_{n} \tau\right) \cos \omega_{n} x, \quad\left(2 \cos \sigma_{n} \tau,-2 \sigma_{n} \sin \sigma_{n} \tau\right) \cos \omega_{n} x, \\
\left(-\cos \sigma_{n} \tau, \sigma_{n} \sin \sigma_{n} \tau\right) r_{n}^{(1)}(x-1) \sin \omega_{n} x+\left(0,-2 \sin \sigma_{n} \tau\right) \cos \omega_{n} x, \\
\left(-\sin \sigma_{n} \tau,-\sigma_{n} \cos \sigma_{n} \tau\right) r_{n}^{(2)}(x-1) \sin \omega_{n} x+\left(0, r_{n}^{(3)} \cos \sigma_{n} \tau\right) \cos \omega_{n} x,
\end{gathered}
$$

где

$$
\begin{aligned}
r_{m}=\left(\lambda_{m, 1}^{(1)}-\lambda_{m, 1}^{(2)}\right) \frac{\left(1+\varkappa \omega_{m}^{2}\right)^{2}}{\omega_{m}}, & r_{n}^{(1)}=2 \sigma_{n} \frac{\left(1+\varkappa \omega_{n}^{2}\right)^{2}}{\omega_{n}}, \\
r_{n}^{(2)}=2 \sigma_{n}\left(\lambda_{3}-\lambda_{4}\right) \frac{\left(1+\varkappa \omega_{n}^{2}\right)^{2}}{\omega_{n}}, & r_{n}^{(3)}=2\left(\lambda_{3}-\lambda_{4}\right) .
\end{aligned}
$$

Остановимся на свойствах коэффициентов (2.56). Предполагаемые в теореме 2.1 условия $\gamma \neq 2 \gamma_{n}, 3 \gamma_{n} / 2$ гарантируют выполнение неравенств $r_{m} \neq 0$, $m \geqslant 1, m \neq n, r_{\infty}=\lim _{m \rightarrow \infty} r_{m} \neq 0$ и $r_{n}^{(2)} \neq 0$ соответственно. А отсюда, в свою очередь, следует, что система (2.54), (2.55) эквивалентна системе

$$
\begin{aligned}
& \frac{1}{\omega_{m}^{2}}(1,0) \cos \omega_{m} x, \quad \frac{1}{\omega_{m}^{2}}(0,1) \cos \omega_{m} x, \quad \frac{1}{\omega_{m}^{2}}(1,0)(x-1) \sin \omega_{m} x, \\
& \frac{1}{\omega_{m}^{2}}(0,1)(x-1) \sin \omega_{m} x, \quad m \geqslant 1,
\end{aligned}
$$

которая образует базис Рисса в $\Sigma(\tau, 0)=E \times E$, где, напомним, $E$ - пространство, введенное в п. 1.2 . 
При $\varepsilon>0$ базисность системы $(2.53)$ в пространстве $\Sigma(\tau, \varepsilon)$ сохраняется, что следует из структуры входящих в (2.34) функций $w_{1, m}, w_{2, m}$. Действительно, так как для любого корня уравнения (2.41) справедливо асимптотическое равенство

$$
2 \lambda_{m, 1}+1=O\left(\frac{1}{\omega_{m}^{3}}\right), \quad m \rightarrow \infty,
$$

то коэффициент перед $B_{m}(x)$ в формуле $(2.36)$ является ограниченным по $m$. Далее, специальный выбор $\lambda_{m, 2}$ обеспечивает ограниченность всех коэффициентов, входящих в $C_{2, m, 0}(x)($ см. $(2.43))$. При этом и сама последовательность $\lambda_{m, 2}$ оказывается ограниченной. И наконец, равномерная по $m$ ограниченность функций $(2.44)$ в метрике $\mathrm{W}_{2}^{2}(0,1)$ - следствие предполагаемых условий (2.45).

Второй этап обоснования алгоритма исследования устойчивости заключается в построении бесконечномерной замены переменных, приводящей исходную задачу (2.23) к некоторой нормальной форме. Для этого нам потребуется линейное пространство $Z$, состоящее из бесконечномерных векторов

$$
\begin{gathered}
z=\left(z_{1}^{(1)}, z_{1}^{(2)}, \bar{z}_{1}^{(1)}, \bar{z}_{1}^{(2)}, \ldots, z_{n-1}^{(1)}, z_{n-1}^{(2)}, \bar{z}_{n-1}^{(1)}, \bar{z}_{n-1}^{(2)}, z_{n}^{(1)}, z_{n}^{(2)}, z_{n}^{(3)}, z_{n}^{(4)},\right. \\
\left.z_{n+1}^{(1)}, z_{n+1}^{(2)}, \bar{z}_{n+1}^{(1)}, \bar{z}_{n+1}^{(2)}, \ldots, z_{m}^{(1)}, z_{m}^{(2)}, \bar{z}_{m}^{(1)}, \bar{z}_{m}^{(2)}, \ldots\right)
\end{gathered}
$$

для которых конечна норма

$$
\|z\|=\left(\sum_{j=1}^{4}\left|z_{n}^{(j)}\right|^{2}+\sum_{\substack{m=1 \\ m \neq n}}^{\infty}\left[\left|z_{m}^{(1)}\right|^{2}+\left|z_{m}^{(2)}\right|^{2}\right]\right)^{1 / 2} .
$$

Здесь все $z_{m}^{(j)}, j=1,2, m \neq n$, комплексные, координаты $z_{n}^{(1)}, z_{n}^{(2)}$ вещественные, а относительно компонент $z_{n}^{(3)}, z_{n}^{(4)}$ предполагаем следующее: $z_{n}^{(3)}, z_{n}^{(4)} \in \mathbb{R}$ при условии вещественности фигурирующих в (2.33) корней $\lambda_{3}, \lambda_{4}$ и $z_{n}^{(3)}, z_{n}^{(4)} \in$ $\mathbb{C}, z_{n}^{(4)}=\bar{z}_{n}^{(3)}$ в противном случае. Подчеркнем, что пространство $Z$ следует рассматривать над полем действительных чисел, так как только в этом случае при умножении его элементов на скаляры снова будут получаться векторы вида (2.57).

Рассмотрим линейный оператор $V(\tau, \varepsilon)$, действующий из $Z$ в $\Sigma(\tau, \varepsilon)$ по правилу

$$
z \in Z \stackrel{V}{\longmapsto} h=\left(h_{1}(x), h_{2}(x)\right) \in \Sigma(\tau, \varepsilon)
$$

где

$$
h=\sum_{j=1}^{4} z_{n}^{(j)} e_{n}^{(j)}+\sum_{\substack{m=1 \\ m \neq n}}^{\infty} \sum_{j=1}^{2}\left[z_{m}^{(j)} e_{m}^{(j)}+\bar{z}_{m}^{(j)} \bar{e}_{m}^{(j)}\right] .
$$

Из явного вида функций $(2.50),(2.52)$ очевидным образом следует ограниченность этого оператора, а также его бесконечная дифференцируемость по $\tau$, $\varepsilon$ в равномерной операторной топологии. Что же касается вопроса об обратимости оператора (2.58), (2.59), то здесь ситуация немного сложнее. Для того чтобы убедиться в наличии требуемого свойства, воспользуемся представлениem

$$
V(\tau, \varepsilon)=V_{0}(\tau, \varepsilon) B_{0},
$$


где операторы $V_{0}: Z \rightarrow \Sigma(\tau, \varepsilon), B_{0}: Z \rightarrow Z$ задаются равенствами

$$
\begin{gathered}
V_{0} z=\sum_{j=1}^{4} z_{n}^{(j)} g_{n}^{(j)}+\sum_{\substack{m=1 \\
m \neq n}}^{\infty} \sum_{j=1}^{2}\left[z_{m}^{(j)} g_{m}^{(j)}+\bar{z}_{m}^{(j)} \bar{g}_{m}^{(j)}\right], \\
B_{0} z=\left(z_{1}^{(1)}+z_{1}^{(2)},-\varepsilon z_{1}^{(2)}, \bar{z}_{1}^{(1)}+\bar{z}_{1}^{(2)},-\varepsilon \bar{z}_{1}^{(2)}, \ldots, z_{n-1}^{(1)}+z_{n-1}^{(2)},-\varepsilon z_{n-1}^{(2)},\right. \\
\bar{z}_{n-1}^{(1)}+\bar{z}_{n-1}^{(2)},-\varepsilon \bar{z}_{n-1}^{(2)}, z_{n}^{(1)}+z_{n}^{(2)},-\varepsilon z_{n}^{(2)}, z_{n}^{(3)}+z_{n}^{(4)},-\varepsilon z_{n}^{(4)}, \\
z_{n+1}^{(1)}+z_{n+1}^{(2)},-\varepsilon z_{n+1}^{(2)}, \bar{z}_{n+1}^{(1)}+\bar{z}_{n+1}^{(2)},-\varepsilon \bar{z}_{n+1}^{(2)}, \ldots, \\
\left.z_{m}^{(1)}+z_{m}^{(2)},-\varepsilon z_{m}^{(2)}, \bar{z}_{m}^{(1)}+\bar{z}_{m}^{(2)},-\varepsilon \bar{z}_{m}^{(2)}, \ldots\right) .
\end{gathered}
$$

Из формулы (2.61) и из установленных выше свойств базисности системы $(2.53)$ заключаем, что оператор $V_{0}(\tau, \varepsilon)$ имеет ограниченный обратный $V_{0}^{-1}$, обладающий теми же свойствами гладкости по $\tau, \varepsilon$, что и $V(\tau, \varepsilon)$. Далее, как показывает непосредственная проверка, оператор (2.62) также обратим, однако норма $B_{0}^{-1}$ имеет порядок $\varepsilon^{-1}$. И наконец, суммируя эти факты, убеждаемся в обратимости исходного оператора $V(\tau, \varepsilon)$ и в справедливости вытекающего из (2.60) равенства

$$
V^{-1}(\tau, \varepsilon)=B_{0}^{-1} V_{0}^{-1}(\tau, \varepsilon) .
$$

На заключительном этапе выполним в уравнении из (2.23) замену переменных, индуцированную оператором (2.58). Для этого сначала, полагая $h_{1}=u$, $h_{2}=\partial u / \partial \tau$, перейдем от указанного уравнения к системе

$$
\left(I-\varkappa \frac{\partial^{2}}{\partial x^{2}}\right) \frac{\partial h}{\partial \tau}=\left(I-\varkappa \frac{\partial^{2}}{\partial x^{2}}\right) D_{1} h+\frac{\partial^{2}}{\partial x^{2}} D_{2} h,
$$

где $h=\left(h_{1}, h_{2}\right) \in \Sigma(\tau, \varepsilon), D_{1} h=\left(h_{2},-\varepsilon h_{2}\right), D_{2} h=\left(0, h_{1}\right)$. Подставим затем в получившуюся систему соотношение

$$
h=V(\tau, \varepsilon) z,
$$

считая, что переменная $z$ дифференцируется по правилу

$$
\frac{d z}{d \tau}=\Lambda_{0}(\varepsilon) z+\varepsilon^{2} \Lambda_{1}(\tau, \varepsilon) z
$$

где $\widetilde{z}=\Lambda_{0}(\varepsilon) z-$ вектор с компонентами

$$
\begin{gathered}
\widetilde{z}_{m}^{(j)}=\left(i \sigma_{m}+\varepsilon \lambda_{m, 1}^{(j)}+\varepsilon^{2} \lambda_{m, 2}\right) z_{m}^{(j)}, \quad j=1,2, \quad m \geqslant 1, \quad m \neq n ; \\
\widetilde{z}_{n}^{(j)}=\varepsilon \lambda_{j} z_{n}^{(j)}, \quad j=1,2,3,4,
\end{gathered}
$$

а $\Lambda_{1}: Z \rightarrow Z$ - некоторый ограниченный линейный оператор, $\left(2 \pi / \sigma_{n}\right)$-периодический по $\tau$ и бесконечно дифференцируемый по совокупности переменных в равномерной топологии. В результате приходим к равенству в пространстве $\mathrm{L}_{2}(0,1) \times \mathrm{L}_{2}(0,1)$ вида

$$
\widetilde{V}(\tau, \varepsilon) \Lambda_{1}(\tau, \varepsilon) z=\varepsilon \Omega(\tau, \varepsilon) z,
$$

где $\widetilde{V}=\left(I-\varkappa \partial^{2} / \partial x^{2}\right) V(\tau, \varepsilon)$, а оператор $\Omega(\tau, \varepsilon): Z \rightarrow \mathrm{L}_{2}(0,1) \times \mathrm{L}_{2}(0,1)$ ограничен равномерно по $\tau, \varepsilon$ и бесконечно дифференцируем по указанным переменным. 
Обращаем внимание, что оператор $\widetilde{V}$, действующий из $Z$ в $\mathrm{L}_{2}(0,1) \times \mathrm{L}_{2}(0,1)$, наследует свойства исходного оператора $V$. В частности, для него остаются в силе представления $(2.60),(2.63)$, в которых оператор $B_{0}$ задается прежним равенством $(2.62)$, а $V_{0}$ заменяется на $\widetilde{V}_{0}=\left(I-\varkappa \partial^{2} / \partial x^{2}\right) V_{0}$. А отсюда следует, что оператор $\Lambda_{1}$, имеющий вид (см. (2.68))

$$
\Lambda_{1}=\varepsilon \widetilde{V}^{-1}(\tau, \varepsilon) \Omega(\tau, \varepsilon),
$$

обладает всеми перечисленными выше свойствами.

Получившаяся система (2.66) и является искомой нормальной формой задачи (2.23). Что же касается ее свойств устойчивости, то они определяются по главной части

$$
\frac{d z}{d \tau}=\Lambda_{0}(\varepsilon) z
$$

а значит, в конечном итоге - по знакам чисел (2.46).

Отметим еще одно свойство системы (2.69), которое потребуется в дальнейшем. С этой целью введем в рассмотрение банахово пространство $C$, состоящее из непрерывных $\left(2 \pi / \sigma_{n}\right)$-периодических по $\tau$ вектор-функций $f(\tau)$ со значениями в $Z$. Обозначим через $C_{0} \subset C$ - подпространство функций, удовлетворяющих дополнительному условию

$$
H(f) \equiv \frac{\sigma_{n}}{2 \pi} \int_{0}^{2 \pi / \sigma_{n}} f_{n}^{(1)}(\tau) d \tau=0,
$$

где $f_{n}^{(1)}$ - соответствующая компонента вектора $f \in Z$ (см. (2.57)). Норму в $C$ определим равенством

$$
\|f\|_{C}=\max _{0 \leqslant \tau \leqslant 2 \pi / \sigma_{n}}\|f(\tau)\|_{Z}
$$

И наконец, рассмотрим неоднородную систему

$$
\frac{d z}{d \tau}=\Lambda_{0}(\varepsilon) z+f(\tau)
$$

с произвольной функцией $f(\tau) \in C_{0}$. Из явного вида оператора $\Lambda_{0}$ (см. (2.67)) очевидным образом следует, что система (2.72) имеет единственное решение $z_{f}(\tau) \in C_{0}$, удовлетворяющее оценке

$$
\left\|\frac{d z_{f}}{d \tau}\right\|_{C}+\left\|z_{f}\right\|_{C} \leqslant \frac{M}{\varepsilon}\|f\|_{C}
$$

с не зависящей от $\varepsilon$ и $f$ постоянной $M>0$.

Итак, преодолена главная трудность, возникающая при обосновании теоремы 2.1, а именно, проделан полный анализ системы в вариациях на приближенном цикле. Доказательство же существования у краевой задачи (1.27) точного периодического решения с построенной в п. 2.1 асимптотикой, как уже отмечалось выше, базируется на стандартных схемах из [9], [11]. Поэтому, опуская технические детали, приведем основные этапы реализации аналитического подхода из [11], который в данном случае является наиболее простым. 
Выполним в (1.27) замену времени $\tau=\left(1+\varepsilon^{2} \delta\right) t$, где $\delta$ - произвольный (подлежащий определению) вещественный параметр, а затем положим в получившейся краевой задаче

$$
u=\varepsilon u_{0}+\varepsilon^{2} u_{1}+\varepsilon^{3} u_{2}+\varepsilon^{2} h_{1}, \quad \frac{\partial u}{\partial \tau}=\varepsilon \frac{\partial u_{0}}{\partial \tau}+\varepsilon^{2} \frac{\partial u_{1}}{\partial \tau}+\varepsilon^{3} \frac{\partial u_{2}}{\partial \tau}+\frac{\varepsilon^{2} h_{2}}{1+\varepsilon^{2} \delta},
$$

где $u_{j}, j=0,1,2,-$ функции из (2.3), в которых учтены соотношения (2.18) и равенства $\eta_{1}=\eta_{2}=0, \delta_{2}=\delta$ (см. (2.8), (2.12)). В результате для $h=\left(h_{1}, h_{2}\right)$ получается аналогичная (2.64) линейная неоднородная система

$$
\left(1+\varepsilon^{2} \delta\right)\left(I-\varkappa \frac{\partial^{2}}{\partial x^{2}}\right) \frac{\partial h}{\partial \tau}-\left(I-\varkappa \frac{\partial^{2}}{\partial x^{2}}\right) D_{1} h-\frac{\partial^{2}}{\partial x^{2}} D_{2} h=\varepsilon^{2} G(\tau, x, \varepsilon, \delta),
$$

где $G$ - некоторая ограниченная в метрике $\mathrm{L}_{2}(0,1) \times \mathrm{L}_{2}(0,1)$ периодическая по $\tau$ вектор-функция.

Напомним, что поскольку граничные условия в (1.27) нелинейны, то и систему (2.75) приходится рассматривать на соответствующем нелинейном многообразии. Для того чтобы избавиться от этой трудности, сделаем сначала в (2.75) аналогичную (1.24) "распрямляющую” замену переменных, переводящую упомянутое нелинейное многообразие в линейное пространство $\Sigma(\tau, \varepsilon)$, а затем выполним замену (2.65). В результате приходим к системе в пространстве $Z$ вида

$$
\frac{d z}{d \tau}=\Lambda_{0}(\varepsilon, \delta) z+\varepsilon F(\tau, z, \varepsilon, \delta)
$$

Здесь оператор $\Lambda_{0}(\varepsilon, \delta)$ отличается от аналогичного линейного оператора $\Lambda_{0}(\varepsilon)$ из (2.66) лишь тем, что фигурирующие в (2.67) постоянные $\lambda_{m, 2}$ зависят от $\delta$. Точнее говоря, для них справедливы формулы (ср. с (2.43))

$$
\lambda_{m, 2}=\frac{i}{2 \sigma_{m}}\left[\lambda_{m, 1}\left(\lambda_{m, 1}+1\right)-2 \delta \sigma_{m}^{2}+\varkappa \omega_{m}^{2}\left(2 \lambda_{m, 1}+1\right)^{2}\right] .
$$

Что же касается $\left(2 \pi / \sigma_{n}\right)$-периодической по $\tau$ вектор-функции $F$ со значениями в $Z$, то она достаточно гладко зависит от всех переменных и удовлетворяет неравенствам

$$
\begin{gathered}
\|F(\tau, 0, \varepsilon, \delta)\|_{C} \leqslant M_{1} \\
\left\|F\left(\tau, z_{1}, \varepsilon, \delta\right)-F\left(\tau, z_{2}, \varepsilon, \delta\right)\right\|_{C} \leqslant M_{2} \varepsilon\left\|z_{1}-z_{2}\right\|_{Z},
\end{gathered}
$$

где $\|\cdot\|_{C}-$ норма $(2.71)$, а положительные постоянные $M_{1}$ и $M_{2}=M_{2}(R)$, $R=\max \left\{\left\|z_{1}\right\|,\left\|z_{2}\right\|\right\}$, не зависят от $\varepsilon$.

Заменим в правой части уравнения (2.76) неоднородность $F$ на

$$
F-H(F) e_{*} \in C_{0},
$$

где $H(\cdot)$ - функционал $(2.70), e_{*}$ - вектор из $Z$, у которого $z_{n}^{(1)}=\bar{z}_{n}^{(1)}=1$, а все остальные компоненты равны нулю. После этого перейдем от (2.76) к интегральному уравнению, обращая оператор $d / d \tau-\Lambda_{0}(\varepsilon, \delta)$. Неравенства $(2.73),(2.77)$ позволяют применить к получившемуся уравнению в пространстве $C_{0}$ принцип сжимающих отображений и определить функцию $z_{*}(\tau, \varepsilon, \delta)$, 
$\left\|z_{*}\right\|_{C} \leqslant M_{3}$, где, как и выше, постоянная $M_{3}>0$ не зависит от $\varepsilon$. Подставляя затем $z=z_{*}(\tau, \varepsilon, \delta)$ в равенство $H(F)=0$, т.е. зануляя сделанную ранее поправку к правой части уравнения $(2.76)$, для $\delta$ получаем уравнение вида

$$
\delta=p(\varepsilon)+\varepsilon \Phi(\varepsilon, \delta)
$$

где функция $p(\varepsilon)$ ограничена.

Можно показать (соответствующий анализ в близкой ситуации проделан, например, в [2]), что функции $\Phi(\varepsilon, \delta), \Phi_{\delta}^{\prime}(\varepsilon, \delta)$ ограничены равномерно по $\varepsilon$ на любом компактном множестве изменения переменной $\delta$. Поэтому, применяя к уравнению (2.78) теорему о неявной функции, однозначно определяем интересующую нас поправку к частоте $\delta=\delta(\varepsilon)$. В итоге, обращая все замены, сделанные при переходе от $(2.74)$ к (2.76), убеждаемся в существовании у исходной задачи (1.27) цикла

$$
u=\varepsilon u_{0}(\tau, x)+\varepsilon^{2} u_{*}(\tau, x, \varepsilon), \quad \frac{d \tau}{d t}=1+\varepsilon^{2} \delta(\varepsilon),
$$

где функция $u_{*}(\tau, x, \varepsilon), u_{*}\left(\tau+2 \pi / \sigma_{n}, x, \varepsilon\right) \equiv u_{*}(\tau, x, \varepsilon)$, и ее производные $\tau$ до второго порядка включительно ограничены равномерно по $\tau, \varepsilon$ в метрике пространства $\mathrm{W}_{2}^{2}(0,1)$. Что же касается свойств устойчивости цикла $(2.79)$, то, как уже было сказано выше, они определяются по уравнению первого приближения (2.69). Теорема 2.1 полностью доказана.

2.3. О существовании и устойчивости инвариантных торов. Для получения более полного представления о динамике краевой задачи (1.27) воспользуемся бесконечномерным вариантом описанного в п. 2.1 алгоритма построения автоколебаний. А именно, в данном случае, обыгрывая существование у линейной краевой задачи (2.1) счетного числа периодических решений (2.2), возможные автоколебательные режимы исходной задачи (1.27) будем искать в виде формального ряда

$$
u=\varepsilon u_{0}(t, \tau, x)+\varepsilon^{2} u_{1}(t, \tau, x)+\varepsilon^{3} u_{2}(t, \tau, x)+\cdots,
$$

где $\tau=\varepsilon t$,

$$
u_{0}=\sum_{n=1}^{\infty}\left[\xi_{n}(\tau) \exp \left(i \sigma_{n} t\right)+\bar{\xi}_{n}(\tau) \exp \left(-i \sigma_{n} t\right)\right] \cos \omega_{n} x,
$$

а подлежащие определению комплексные амплитуды $\xi_{n}$ таковы, что сходится ряд с общим членом $\omega_{n}^{4}\left|\xi_{n}\right|^{2}$ (в этом случае $u_{0} \in \mathrm{W}_{2}^{2}$ по переменной $x$ ).

Подставляя (2.80), (2.81) в (1.27) и приравнивая коэффициенты при $\varepsilon^{2}$, для $u_{1}$ получим аналогичную (2.5) краевую задачу

$$
\begin{gathered}
\Pi\left(u_{1}\right)=-\left(2 \frac{\partial^{2}}{\partial t \partial \tau}+\frac{\partial}{\partial t}\right)\left(u_{0}-\varkappa \frac{\partial^{2} u_{0}}{\partial x^{2}}\right), \\
\left.\frac{\partial u_{1}}{\partial x}\right|_{x=1}=0,\left.\quad u_{1}\right|_{x=0}+\left.u_{1}\right|_{x=1}=0,
\end{gathered}
$$

где П - оператор из (2.1). Ее решение будем искать в виде формального тригонометрического ряда той же структуры, что и неоднородность. На этом пути 
приходим к равенству

$$
\begin{gathered}
u_{1}=\sum_{n=1}^{\infty} \sigma_{n} \frac{\left(1+\varkappa \omega_{n}^{2}\right)^{2}}{\omega_{n}}\left[\left(\xi_{n}+2 \dot{\xi}_{n}\right) B_{n}(x) \exp \left(i \sigma_{n} t\right)\right. \\
\left.+\left(\bar{\xi}_{n}+2 \dot{\bar{\xi}}_{n}\right) \bar{B}_{n}(x) \exp \left(-i \sigma_{n} t\right)\right]
\end{gathered}
$$

где точка - дифференцирование по $\tau, B_{n}$ - функции (2.9).

После приравнивания коэффициентов при $\varepsilon^{3}$ для $u_{2}$ имеем дело с аналогичной (2.82) линейной неоднородной краевой задачей

$$
\begin{aligned}
\Pi\left(u_{2}\right)= & -\left(\frac{\partial^{2}}{\partial \tau^{2}}+\frac{\partial}{\partial \tau}\right)\left(u_{0}-\varkappa \frac{\partial^{2} u_{0}}{\partial x^{2}}\right)-\left(2 \frac{\partial^{2}}{\partial t \partial \tau}+\frac{\partial}{\partial t}\right)\left(u_{1}-\varkappa \frac{\partial^{2} u_{1}}{\partial x^{2}}\right), \\
& \left.\frac{\partial u_{2}}{\partial x}\right|_{x=1}=0,\left.\quad u_{2}\right|_{x=0}+\left.u_{2}\right|_{x=1}=\left.\left(-\gamma u_{0}+u_{0}^{3}\right)\right|_{x=1} .
\end{aligned}
$$

Как и в предыдущем случае, ее решение ищем в том же виде, что и соответствующие неоднородности, т.е. в виде тригонометрического ряда, состоящего из гармоник

$$
\exp \left[i\left(k_{1} \sigma_{n_{1}}+k_{2} \sigma_{n_{2}}+k_{3} \sigma_{n_{3}}\right) t\right],
$$

где $n_{j}, j=1,2,3,-$ произвольные натуральные числа, а $\left(k_{1}, k_{2}, k_{3}\right)$ - произвольный целочисленный вектор, удовлетворяющий равенству $\left|k_{1}\right|+\left|k_{2}\right|+\left|k_{3}\right|=1$ или $\left|k_{1}\right|+\left|k_{2}\right|+\left|k_{3}\right|=3$. В результате для коэффициентов функции $u_{2}$ при гармониках (2.84) получаются линейные неоднородные краевые задачи, которые в случае $\left|k_{1}\right|+\left|k_{2}\right|+\left|k_{3}\right|=3$ при некоторой общности положения, связанной с выбором параметра $\varkappa$, однозначно разрешимы. Что же касается случая $\left|k_{1}\right|+\left|k_{2}\right|+\left|k_{3}\right|=1$, то здесь для коэффициентов $A_{n}(x), \bar{A}_{n}(x)$ при гармониках $\exp \left( \pm i \sigma_{n} t\right), n \geqslant 1$, имеем вырожденные краевые задачи:

$$
A_{n}^{\prime \prime}+\omega_{n}^{2} A_{n}=f_{n}(x, \tau),\left.\quad A_{n}^{\prime}\right|_{x=1}=0,\left.\quad A_{n}\right|_{x=0}+\left.A_{n}\right|_{x=1}=\psi_{n},
$$

где штрих - дифференцирование по $x$,

$$
\begin{aligned}
f_{n}= & \left(\ddot{\xi}_{n}+\dot{\xi}_{n}\right)\left(1+\varkappa \omega_{n}^{2}\right)^{2} \cos \omega_{n} x \\
& +i \sigma_{n}^{2} \frac{\left(1+\varkappa \omega_{n}^{2}\right)^{3}}{\omega_{n}}\left(4 \ddot{\xi}_{n}+4 \dot{\xi}_{n}+\xi_{n}\right)\left(B_{n}(x)-\varkappa B_{n}^{\prime \prime}(x)\right), \\
\psi_{n}= & \left(\gamma-3\left|\xi_{n}\right|^{2}-6 \sum_{\substack{m=1 \\
m \neq n}}^{\infty}\left|\xi_{m}\right|^{2}\right) \xi_{n},
\end{aligned}
$$

а переменная $\tau$ рассматривается как параметр.

Условия разрешимости краевых задач (2.85), имеющие вид (2.15), будем рассматривать как уравнения для отыскания амплитуд $\xi_{n}$. Несложный анализ указанных условий с учетом формул $(2.86),(2.87)$ приводит к счетной системе обыкновенных дифференциальных уравнений

$$
\left(1+\varkappa \omega_{n}^{2}\right)^{3}\left(\ddot{\xi}_{n}+\dot{\xi}_{n}+\frac{1}{4} \xi_{n}\right)=2\left(\gamma-3\left|\xi_{n}\right|^{2}-6 \sum_{\substack{m=1 \\ m \neq n}}^{\infty}\left|\xi_{m}\right|^{2}\right) \xi_{n}, \quad n \geqslant 1,
$$


которую, следуя [12], будем называть квазинормальной формой исходной краевой задачи (1.27). Подобное название в данном случае оправдано тем, что заведомо не существует корректной бесконечномерной замены переменных, приводящей задачу (1.27) к виду (2.88). Действительно, условие $\sum_{n} \omega_{n}^{4}\left|\xi_{n}\right|^{2}<\infty$ обеспечивает сходимость лишь ряда (2.81), в то время как ряды для более старших коэффициентов $u_{j}, j \geqslant 1$, из (2.80) в общем случае расходятся (см., например, (2.83)).

Несмотря на все вышесказанное, квазинормальная форма (2.88) содержит определенную информацию об аттракторах краевой задачи (1.27). Для того чтобы сформулировать соответствующий строгий результат, фиксируем произвольно набор натуральных чисел

$$
n_{1}<n_{2}<\cdots<n_{k}, \quad k \geqslant 2,
$$

и рассмотрим линейную систему для отыскания $\eta_{j}, j=1, \ldots, k$ :

$$
3 \eta_{j}+6 \sum_{\substack{s=1 \\ s \neq j}}^{k} \eta_{s}=\Delta_{j}, \quad j=1, \ldots, k,
$$

где $\Delta_{j}=\gamma-\gamma_{n_{j}}$, а постоянные $\gamma_{n}$, напомним, определены равенствами (1.33). Предположим, что при каждом $j=1, \ldots, k$ выполняется условие

$$
\Delta_{j}<\frac{2}{2 k-1} \sum_{s=1}^{k} \Delta_{s}
$$

Тогда, очевидно, система (2.90) имеет единственное решение

$$
\eta_{j}=\frac{1}{3}\left(\frac{2}{2 k-1} \sum_{s=1}^{k} \Delta_{s}-\Delta_{j}\right)>0, \quad j=1, \ldots, k,
$$

а значит, у квазинормальной формы (2.88) существует $k$-мерное инвариантное тороидальное многообразие:

$$
\begin{gathered}
\xi_{n_{j}}=\sqrt{\eta_{j}} \exp \left(i \alpha_{j}\right), \quad \alpha_{j}=\text { const } \in \mathbb{R}, \quad j=1, \ldots, k ; \\
\xi_{n}=0 \quad \text { при } \quad n \neq n_{j} .
\end{gathered}
$$

Для исследования устойчивости получившегося многообразия линеаризуем систему (2.88), дополненную уравнениями для $\bar{\xi}_{n}, n \geqslant 1$, на произвольном состоянии равновесия из семейства (2.92). В итоге убеждаемся, что соответствующая система в вариациях имеет блочно-диагональную структуру. Точнее говоря, она распадается на счетное число скалярных уравнений

$$
\left(1+\varkappa \omega_{m}^{2}\right)^{3}\left(\ddot{h}_{m}+\dot{h}_{m}\right)=2 R_{m} h_{m}, \quad m \geqslant 1, \quad m \neq n_{j}, \quad j=1, \ldots, k,
$$

где $R_{m}=\gamma-\gamma_{m}-6 \sum_{s=1}^{k} \eta_{s}$, и конечномерную систему

$$
\begin{gathered}
\ddot{h}_{n_{j}}+\dot{h}_{n_{j}}+\frac{6}{\left(1+\varkappa \omega_{n_{j}}^{2}\right)^{3}}\left[\eta_{j}\left(h_{n_{j}}+\bar{h}_{n_{j}}\right)+2 \sum_{\substack{s=1 \\
s \neq j}}^{k} \eta_{s}\left(h_{n_{s}}+\bar{h}_{n_{s}}\right)\right]=0, \\
j=1, \ldots, k .
\end{gathered}
$$


Отметим, что система (2.94) имеет $k$ нулевых характеристических показателей и $k$ показателей, равных -1 , которым отвечают соответственно решения c компонентами

$$
\begin{aligned}
& h_{n_{r}}=i, \quad h_{n_{s}}=0, \quad s=1, \ldots, k, \quad s \neq r ; \\
& h_{n_{r}}=i \exp (-\tau), \quad h_{n_{s}}=0, \quad s=1, \ldots, k, \quad s \neq r,
\end{aligned}
$$

где $1 \leqslant r \leqslant k$. Для отыскания же остальных ее характеристических показателей сначала будем считать $h_{n_{j}}$ и $\bar{h}_{n_{j}}$ независимыми комплексными переменными, причем во избежание недоразумений обозначим $\bar{h}_{n_{j}}$ через $h_{n_{j}}^{*}$. После этого возможные решения Ляпунова-Флоке получившейся линейной системы для $h_{n_{j}}, h_{n_{j}}^{*}$ ищем в виде

$$
h_{n_{j}}=y_{j} \exp (\lambda t), \quad h_{n_{j}}^{*}=y_{j} \exp (\lambda t), \quad j=1, \ldots, k .
$$

В результате для $y_{j}$ приходим к линейной алгебраической системе

$$
\eta_{j} y_{j}=\frac{24 \eta_{j}}{12 \eta_{j}-\left(1+\varkappa \omega_{n_{j}}^{2}\right)^{3} \mu} \sum_{s=1}^{k} \eta_{s} y_{s}, \quad j=1, \ldots, k
$$

где $\mu=\lambda^{2}+\lambda$. Заметим, что у получившейся системы существуют нетривиальные решения в том и только том случае, когда $\mu$ является корнем уравнения

$$
\sum_{j=1}^{k} \frac{24 \eta_{j}}{12 \eta_{j}-\left(1+\varkappa \omega_{n_{j}}^{2}\right)^{3} \mu}=1
$$

Нетрудно увидеть, что уравнение (2.95) имеет единственный отрицательный корень $\mu=\mu_{0}$ и $k-1$ положительный корень

$$
\mu_{1}, \mu_{2}, \ldots, \mu_{k-1}
$$

которые в общем случае являются различными. Что же касается интересующих нас характеристических показателей $\lambda$, то они определяются из квадратных уравнений

$$
\lambda^{2}+\lambda-\mu_{j}=0, \quad j=0, \ldots, k-1 .
$$

Суммируя проделанные построения и опираясь на общие утверждения монографии [9] (см. [9; гл. 1]), приходим к следующему утверждению.

ТЕОРЕма 2.2. Пусть выполнены неравенства (2.91) и различны все корни (2.96). Пусть, далее, не обращаются в нуль фигурирующие в (2.93) числа $R_{m}$ и количество положительных среди них равно $m_{0}$. Тогда при некоторой дополнительной общности положения, связанной с параметром,$~ u$ при всех достаточно малых $\varepsilon>0$ краевая задача (1.27) имеет дихотомичный $k$-мерныц инвариантный тор с размерностью неустойчивого многообразия, равной $2 m_{0}+k$. Асимптотика решений на этом торе вычисляется в соответствии с формулами (2.80), (2.81), в которых необходимо учесть равенства (2.92). 
Следует отметить, что для любого фиксированного набора индексов (2.89) выполнения условий (2.91) и неравенств $R_{m}<0$ всегда можно добиться за счет подходящего увеличения параметра $\gamma$. А это означает, что краевая задача (1.27) может иметь инвариантные торы любых конечных размерностей. Однако все они заведомо неустойчивы, так как у уравнений (2.97) при $j=$ $1, \ldots, k-1$ существуют положительные корни.

\section{§ 3. Развитая буферность}

3.1. Алгоритмическая часть. Как отмечено в п. 2.1, один из механизмов неограниченного накапливания устойчивых циклов у краевой задачи (1.27) заключается в увеличении $\gamma$ при фиксированных прочих параметрах. Однако попутно происходит увеличение и амплитуд указанных циклов. Например, при $\gamma \sim \varepsilon^{-1}$ их амплитуды согласно формулам $(2.3),(2.4),(2.18)$ должны стать величинами порядка $\sqrt{\varepsilon}$. А это значит, что при строгом рассмотрении вопросов о существовании и устойчивости таких циклов мы заведомо выходим за рамки применимости теоремы 2.1. Для получения же аналога этой теоремы в случае $\gamma \sim \varepsilon^{-1}$ требуется надлежащая модификация алгоритмов из п. 2.1, которая и предлагается ниже.

Итак, анализу подлежит краевая задача

$$
\begin{gathered}
\frac{\partial^{2}}{\partial t^{2}}\left(u-\varkappa \frac{\partial^{2} u}{\partial x^{2}}\right)+\varepsilon \frac{\partial}{\partial t}\left(u-\varkappa \frac{\partial^{2} u}{\partial x^{2}}\right)=\frac{\partial^{2} u}{\partial x^{2}}, \\
\left.\frac{\partial u}{\partial x}\right|_{x=1}=0,\left.\quad u\right|_{x=0}+\left.(1+\varepsilon \gamma) u\right|_{x=1}-\left.u^{3}\right|_{x=1}=0,
\end{gathered}
$$

где $0<\varepsilon \ll 1, \gamma=$ const $>0$, получающаяся из (1.27) при замене $\varepsilon \gamma$ на $\gamma$. Далее, фиксируем произвольно натуральное $n$. Согласно вышесказанному асимптотику ее цикла с частотой, близкой к $\sigma_{n}$, будем строить в виде рядов по четным и нечетным степеням $\sqrt{\varepsilon}$ соответственно:

$\tau=\left(1+\varepsilon \delta_{1}+\varepsilon^{2} \delta_{2}+\cdots\right) t, \quad u=\sqrt{\varepsilon} u_{0}(\tau, x)+\varepsilon^{3 / 2} u_{1}(\tau, x)+\varepsilon^{5 / 2} u_{2}(\tau, x)+\cdots$,

где по-прежнему $u_{0}(\tau, x)$ задается формулой $(2.4)$ и все коэффициенты $u_{j}$, $j \geqslant 1$, периодичны по $\tau$ с периодом $2 \pi / \sigma_{n}$.

Действуя в соответствии с изложенной в п. 2.1 схемой, для $u_{1}$ имеем краевую задачу вида (2.5) с неоднородностями

$$
f_{1}=-2 \delta_{1} \frac{\partial^{2}}{\partial \tau^{2}} \mathscr{L} u_{0}-\frac{\partial}{\partial \tau} \mathscr{L} u_{0}, \quad \psi_{1}=-\left.\left(\gamma u_{0}+u_{0}^{3}\right)\right|_{x=1},
$$

где, напомним, $\mathscr{L} u=u-\varkappa \partial^{2} u / \partial x^{2}$. А отсюда из структуры $u_{0}$ заключаем, что $u_{1}$ следует искать в виде

$$
\begin{aligned}
u_{1}=A_{1,1}(x) \exp \left(i \sigma_{n} \tau\right)+\bar{A}_{1,1}(x) \exp \left(-i \sigma_{n} \tau\right) \\
\quad+A_{1,3}(x) \exp \left(3 i \sigma_{n} \tau\right)+\bar{A}_{1,3}(x) \exp \left(-3 i \sigma_{n} \tau\right) .
\end{aligned}
$$

На этом пути сначала из условия разрешимости вырожденной краевой задачи для $A_{1,1}$ определяем фигурирующую в $u_{0}$ амплитуду

$$
\xi=\sqrt{\frac{\gamma}{3}},
$$


а затем находим и функцию $A_{1,1}$. Интересно отметить, что для $A_{1,1}$ получается в точности формула (2.8), в которой $\xi$ задается равенством $(3.4)$, а $\eta_{1}$ - пока произвольный вещественный параметр. Что же касается функции $A_{1,3}$, то для нее при выполнении условий (2.20) справедливы равенства (2.21), в которых также надо учесть соотношение (3.4).

На следующем шаге принципиально новых моментов не возникает. Действительно, здесь получается краевая задача (2.5), в которой для неоднородности $f_{2}$ сохраняется прежняя формула из (2.10), а $\psi_{2}$ определяется равенством

$$
\psi_{2}=\left.\left(-\gamma u_{1}+3 u_{0}^{2} u_{1}\right)\right|_{x=1} .
$$

Подставляя в формулу (3.5) соотношение (3.3), убеждаемся, что функция $u_{2}$ должна иметь вид

$$
u_{2}=\sum_{k=1}^{3}\left[A_{2,2 k-1}(x) \exp \left(i(2 k-1) \sigma_{n} \tau\right)+\bar{A}_{2,2 k-1}(x) \exp \left(-i(2 k-1) \sigma_{n} \tau\right)\right] .
$$

Таким образом, как и выше, проблема сводится к отысканию фигурирующих в (3.6) коэффициентов $A_{2, j}, j=1,3,5$, из соответствующих линейных неоднородных краевых задач.

В случае $A_{2,1}$ имеем дело с аналогичной (2.13) вырожденной краевой задачей, разрешимости которой добиваемся за счет выбора свободных параметров $\eta_{1}, \delta_{1}$. Несложные вычисления показывают, что

$$
\delta_{1}=0, \quad \eta_{1}=\frac{c}{2}-\frac{\gamma_{n}}{6 \xi},
$$

где $\gamma_{n}, c$ - постоянные (1.33), (2.21). В случае же $A_{2,3}$ и $A_{2,5}$ при условиях $(2.20)$ и при аналогичных условиях нерезонансности

$$
5 \sigma_{n} \neq \sigma_{m}, \quad 5 \sigma_{n} \neq \frac{1}{\sqrt{\varkappa}}, \quad m \geqslant 5 n-1,
$$

получаются невырожденные краевые задачи. Всюду ниже упомянутые условия считаем выполненными, а явные формулы для $A_{2, j}, j=1,3,5$, опустим, поскольку в дальнейшем они не потребуются.

Как установлено в $\S 2$, для доказательства существования у краевой задачи (3.1) цикла с построенной выше главной асимптотикой следует еще разобраться со свойствами устойчивости аналогичной (2.23) линейной краевой задачи

$$
\begin{gathered}
\frac{\partial^{2}}{\partial \tau^{2}}\left(u-\varkappa \frac{\partial^{2} u}{\partial x^{2}}\right)+\varepsilon \frac{\partial}{\partial \tau}\left(u-\varkappa \frac{\partial^{2} u}{\partial x^{2}}\right)=\frac{\partial^{2} u}{\partial x^{2}} \\
\left.\frac{\partial u}{\partial x}\right|_{x=1}=0,\left.\quad u\right|_{x=0}+\left.u\right|_{x=1}+\left.\left(\varepsilon \Delta_{1}(\tau)+\varepsilon^{2} \Delta_{2}(\tau)\right) u\right|_{x=1}=0
\end{gathered}
$$

где $\Delta_{1}=\gamma-3 u_{0}^{2}(\tau, 1), \Delta_{2}=-6 u_{0}(\tau, 1) u_{1}(\tau, 1)$, а $u_{0}, u_{1}-$ функции из $(3.2)$. В связи с этим, опуская некоторые технические моменты, приведем алгоритмы асимптотического вычисления ее решений Ляпунова-Флоке. 
Как и в п. 2.1, начнем с расчета характеристических показателей краевой задачи (3.9) на частоте $\sigma_{n}$. Для этого подставим в (3.9) аналогичное (2.24) соотношение

$$
\begin{gathered}
u=\left[w_{0, n}(\tau, x)+\sqrt{\varepsilon} w_{1, n}(\tau, x)+\varepsilon w_{2, n}(\tau, x)+\varepsilon^{3 / 2} w_{3, n}(\tau, x)\right. \\
\left.+\varepsilon^{2} w_{4, n}(\tau, x)\right] \exp \left[\left(\sqrt{\varepsilon} \lambda_{1}+\varepsilon \lambda_{2}+\varepsilon^{3 / 2} \lambda_{3}\right) \tau\right] .
\end{gathered}
$$

Здесь, как и раньше, все функции являются тригонометрическими полиномами переменной $\sigma_{n} \tau$, причем для $w_{0, n}$ сохраняется формула $(2.25)$, а комплексные постоянные $\lambda_{1}, \lambda_{2}, \lambda_{3}, a_{1}, a_{2}$ подлежат определению.

Приравнивая последовательно коэффициенты при одинаковых степенях $\varepsilon$, для функций $w_{j, n}, j=1,2,3,4$, из (3.10) приходим к серии линейных неоднородных краевых задач вида

$$
\Pi\left(w_{j, n}\right)=f_{j}(\tau, x),\left.\quad \frac{\partial w_{j, n}}{\partial x}\right|_{x=1}=0,\left.\quad w_{j, n}\right|_{x=0}+\left.w_{j, n}\right|_{x=1}=\psi_{j}(\tau),
$$

где оператор П определен в (2.5). Их решения ищем в классе тригонометрических полиномов той же структуры, что и неоднородности. Например, при $j=1$ имеем

$$
f_{1}=-2 \lambda_{1} \frac{\partial}{\partial \tau} \mathscr{L} w_{0, n}, \quad \psi_{1}=0,
$$

а значит, для $w_{1, n}$ справедливы формулы

$$
\begin{gathered}
w_{1, n}=C_{1, n, 1}^{+}(x) \exp \left(i \sigma_{n} \tau\right)+C_{1, n, 1}^{-}(x) \exp \left(-i \sigma_{n} \tau\right), \\
C_{1, n, 1}^{+}=a_{1} W_{0}(x)+b_{1,1} \cos \omega_{n} x, \quad C_{1, n, 1}^{-}=-a_{2} W_{0}(x)+b_{1,2} \cos \omega_{n} x, \\
W_{0}(x)=2 \lambda_{1} \frac{\left(1+\varkappa \omega_{n}^{2}\right)^{2}}{\omega_{n}} \sigma_{n} B_{n}(x),
\end{gathered}
$$

где $b_{1,1}, b_{1,2}$ - произвольные комплексные постоянные.

При $j=2$ в силу равенств

$$
f_{2}=-2 \lambda_{1} \frac{\partial}{\partial \tau} \mathscr{L} w_{1, n}-\lambda_{1}^{2} \mathscr{L} w_{0, n}-\left(2 \lambda_{2}+1\right) \frac{\partial}{\partial \tau} \mathscr{L} w_{0, n}, \quad \psi_{2}=-\left.\Delta_{1}(\tau) w_{0, n}\right|_{x=1}
$$

решение $w_{2, n}$ задачи (3.11) следует искать в виде (2.29). В результате из условий разрешимости краевых задач для $C_{2, n, 1}^{ \pm}$получаем аналогичную $(2.30)$ систему

$$
-3 \xi^{2}\left(a_{1}+a_{2}\right)=4 \lambda_{1}^{2} \gamma_{n} a_{1}, \quad-3 \xi^{2}\left(a_{1}+a_{2}\right)=4 \lambda_{1}^{2} \gamma_{n} a_{2}
$$

для $a_{1}, a_{2}$. Заметим, далее, что эта система допускает нетривиальные решения только в случаях

$$
\lambda_{1}=0, \quad \lambda_{1}= \pm i \xi \sqrt{\frac{3}{2 \gamma_{n}}},
$$

причем первому из них отвечают значения $a_{1}=i, a_{2}=-i$, а двум другим значения $a_{1}=a_{2}=1$.

Итак, пусть параметры $\lambda_{1}, a_{1}, a_{2}$ выбраны по указанному выше правилу. Тогда все фигурирующие в $w_{2, n}$ коэффициенты $C_{2, n, 1}^{ \pm}, C_{2, n, 3}^{ \pm}$могут быть найдены. Проводя соответствующие вычисления, убеждаемся в справедливости 
равенств

$$
\begin{aligned}
& C_{2, n, 1}^{+}=- 3 \xi^{2}\left(a_{1}+a_{2}\right)\left[(x-1)^{2} \cos \omega_{n} x-\frac{1}{\omega_{n}}(x-1) \sin \omega_{n} x\right] \\
&+a_{1} \frac{\left(1+\varkappa \omega_{n}^{2}\right)^{2}}{\omega_{n}}\left[\sigma_{n}\left(2 \lambda_{2}+1\right)-i \lambda_{1}^{2}\left(1+4 \varkappa \omega_{n}^{2}\right)\right] B_{n}(x) \\
&+2 \lambda_{1} b_{1,1} \sigma_{n} \frac{\left(1+\varkappa \omega_{n}^{2}\right)^{2}}{\omega_{n}} B_{n}(x)+b_{2,1} \cos \omega_{n} x, \\
& C_{2, n, 1}^{-}=- 3 \xi^{2}\left(a_{1}+a_{2}\right)\left[(x-1)^{2} \cos \omega_{n} x-\frac{1}{\omega_{n}}(x-1) \sin \omega_{n} x\right] \\
&-a_{2} \frac{\left(1+\varkappa \omega_{n}^{2}\right)^{2}}{\omega_{n}}\left[\sigma_{n}\left(2 \lambda_{2}+1\right)+i \lambda_{1}^{2}\left(1+4 \varkappa \omega_{n}^{2}\right)\right] B_{n}(x) \\
&-2 \lambda_{1} b_{1,2} \sigma_{n} \frac{\left(1+\varkappa \omega_{n}^{2}\right)^{2}}{\omega_{n}} B_{n}(x)+b_{2,2} \cos \omega_{n} x, \\
& C_{2, n, 3}^{+}=\frac{3 a_{1} c}{\xi} \cos \frac{3 \omega_{n}(x-1)}{\sqrt{1-8 \varkappa \omega_{n}^{2}}}, \quad C_{2, n, 3}^{-}=\frac{3 a_{2} c}{\xi} \cos \frac{3 \omega_{n}(x-1)}{\sqrt{1-8 \varkappa \omega_{n}^{2}}},
\end{aligned}
$$

где, как и в (3.12), $b_{2,1}, b_{2,2}$ - произвольные комплексные постоянные, а константа $c$ заимствована из (2.21).

При $j=3$ сталкиваемся с той же ситуацией, что и выше. Действительно, учитывая в равенствах

$$
\begin{aligned}
f_{3}=- & 2 \lambda_{3} \frac{\partial}{\partial \tau} \mathscr{L} w_{0, n}-\lambda_{1}\left(2 \lambda_{2}+1\right) \mathscr{L} w_{0, n}-\left(2 \lambda_{2}+1\right) \frac{\partial}{\partial \tau} \mathscr{L} w_{1, n} \\
& -\lambda_{1}^{2} \mathscr{L} w_{1, n}-2 \lambda_{1} \frac{\partial}{\partial \tau} \mathscr{L} w_{2, n}, \quad \psi_{3}=-\left.\Delta_{1}(\tau) w_{1, n}\right|_{x=1}
\end{aligned}
$$

явный вид уже найденных функций $w_{1, n}, w_{2, n}$, убеждаемся, что $w_{3, n}$ - комбинация первых и третьих гармоник. Далее, для коэффициентов из $w_{3, n}$ при первых гармониках получаются, как обычно, вырожденные краевые задачи. Выполнения же условий их разрешимости добиваемся за счет подходящего выбора постоянных $b_{1,1}, b_{1,2}$ (см. (3.12)). На этом пути для указанных постоянных получается аналогичная (3.13) линейная система

$$
-3 \xi^{2}\left(b_{1,1}+b_{1,2}\right)-4 \lambda_{1}^{2} \gamma_{n} b_{1,1}=G_{1}, \quad-3 \xi^{2}\left(b_{1,1}+b_{1,2}\right)-4 \lambda_{1}^{2} \gamma_{n} b_{1,2}=G_{2},
$$

где неоднородности $G_{1}, G_{2}$ имеют вид

$$
G_{j}=\frac{1}{2} a_{j} \lambda_{1}\left(1+\varkappa \omega_{n}^{2}\right)^{3}\left(2 \lambda_{2}+1\right)+G_{j}^{0}, \quad j=1,2 .
$$

Явные выражения для слагаемых $G_{j}^{0}$ из (3.19) опустим. Отметим только, что они не зависят от $\lambda_{2}$ и при $a_{1}=a_{2}$ удовлетворяют соотношению

$$
G_{1}^{0}=-G_{2}^{0}
$$

Дальнейший анализ существенно зависит от выбора показателя $\lambda_{1}$. Предположим сначала, что в качестве $\lambda_{1}$ взято одно из ненулевых значений (3.14), которому, напомним, соответствуют равенства $a_{1}=a_{2}=1$. Тогда из условия 
разрешимости вырожденной системы (3.18), имеющего вид $G_{1}+G_{2}=0$, в силу (3.19), (3.20) находим $\lambda_{2}=-1 / 2$. Что же касается функции $w_{4, n}$, то она является линейной комбинацией нечетных гармоник переменной $\sigma_{n} \tau$ до пятого порядка включительно, а разрешимости краевых задач для ее коэффициентов при первых гармониках добиваемся за счет фигурирующих в (3.15), (3.16) постоянных $b_{2,1}, b_{2,2}$ и параметра $\lambda_{3}$ (для $b_{2,1}, b_{2,2}$ получается аналогичная (3.18) вырожденная неоднородная линейная система, а постоянная $\lambda_{3}$ обеспечивает ее разрешимость). Остальные же коэффициенты как в $w_{3, n}$, так и в $w_{4, n}$ определяются однозначно в силу условий (2.20), (3.8).

Обратимся затем к случаю $\lambda_{1}=0$, в котором $w_{1, n}=w_{3, n}=0, \lambda_{3}=0, a_{1}=i$, $a_{2}=-i$, а для неоднородностей в краевой задаче (3.11) при $j=4$ справедливы равенства

$$
\begin{aligned}
& f_{4}=-\left(2 \lambda_{2}+1\right) \frac{\partial}{\partial \tau} \mathscr{L} w_{2, n}-\lambda_{2}\left(\lambda_{2}+1\right) \mathscr{L} w_{0, n}, \\
& \psi_{4}=-\left.\Delta_{1} w_{2, n}\right|_{x=1}-\left.\Delta_{2} w_{0, n}\right|_{x=1} .
\end{aligned}
$$

Учитывая в (3.21) явные выражения для $w_{0, n}, w_{2, n}, u_{0}, u_{1}$ (см. (2.4), (2.25), $(3.3),(3.7),(3.15)-(3.17))$, приходим к выводу, что функцию $w_{4, n}$ следует искать в виде

$$
w_{4, n}=\sum_{k=1}^{3}\left[C_{4, n, 2 k-1}^{+}(x) \exp \left(i(2 k-1) \sigma_{n} \tau\right)+C_{4, n, 2 k-1}^{-}(x) \exp \left(-i(2 k-1) \sigma_{n} \tau\right)\right] .
$$

При этом для коэффициентов $C_{4, n, 3}^{ \pm}, C_{4, n, 5}^{ \pm}$в силу условий $(2.20),(3.8)$ получаются невырожденные линейные неоднородные краевые задачи, а из условий разрешимости краевых задач для $C_{4, n, 1}^{ \pm}$имеем систему

$3 \xi^{2}\left(b_{2,1}+b_{2,2}\right)=i \gamma_{n}\left(1-\left(2 \lambda_{2}+1\right)^{2}\right), \quad 3 \xi^{2}\left(b_{2,1}+b_{2,2}\right)=-i \gamma_{n}\left(1-\left(2 \lambda_{2}+1\right)^{2}\right)$.

Остается добавить, что последняя разрешима только в двух случаях: $\lambda_{2}=0$ или $\lambda_{2}=-1$.

Приступим теперь к расчету характеристических показателей задачи (3.9) на частотах $\sigma_{m}, m \neq n$. С этой целью подставим в нее аналогичное $(2.34)$ выражение

$$
\begin{aligned}
u=[ & \left.\cos \omega_{m} x+\sqrt{\varepsilon} w_{1, m}+\varepsilon w_{2, m}+\varepsilon^{3 / 2} w_{3, m}+\varepsilon^{2} w_{4, m}\right] \\
& \times \exp \left[\left(i \sigma_{m}+\sqrt{\varepsilon} \lambda_{m, 1}+\varepsilon \lambda_{m, 2}+\varepsilon^{3 / 2} \lambda_{m, 3}+\varepsilon^{2} \lambda_{m, 4}\right) \tau\right],
\end{aligned}
$$

где, как обычно, функции $w_{j, m}, j=1,2,3,4$, являющиеся тригонометрическими полиномами переменной $\sigma_{n} \tau$, подлежат определению вместе с постоянными $\lambda_{m, j}$. В результате после приравнивания коэффициентов при одинаковых степенях $\varepsilon$ приходим к серии линейных неоднородных краевых задач

$$
\Pi_{m}\left(w_{j, m}\right)=f_{j, m}(\tau, x),\left.\quad \frac{\partial w_{j, m}}{\partial x}\right|_{x=1}=0,\left.\quad w_{j, m}\right|_{x=0}+\left.w_{j, m}\right|_{x=1}=\psi_{j, m},
$$

где $\Pi_{m}$ - оператор из (2.37).

Несложный подсчет показывает, что для $w_{1, m}$ справедлива аналогичная (2.36) формула

$$
w_{1, m}=2 \lambda_{m, 1} \sigma_{m} \frac{\left(1+\varkappa \omega_{m}^{2}\right)^{2}}{\omega_{m}} B_{m}(x)
$$


а неоднородности в краевой задаче (3.23) при $j=2$ имеют вид

$$
\begin{gathered}
f_{2, m}=-\left(1+\varkappa \omega_{m}^{2}\right)\left(\lambda_{m, 1}^{2}+i \sigma_{m}\left(2 \lambda_{m, 2}+1\right)\right) \cos \omega_{m} x-2 i \sigma_{m} \lambda_{m, 1} \mathscr{L} w_{1, m} \\
\psi_{2, m}=\Delta_{1}(\tau) .
\end{gathered}
$$

Далее, из (3.24), (3.25) следует, что $w_{2, m}$ задается прежним соотношением (2.38), причем для коэффициента $C_{2, m, 0}$ получается аналогичная (2.39) вырожденная краевая задача. Разрешимости последней добиваемся за счет выбора параметра $\lambda_{m, 1}$. Проводя соответствующие вычисления (см. аналогичное место в п. 2.1), получаем равенства

$$
\begin{gathered}
\lambda_{m, 1}= \pm i \xi \sqrt{\frac{3}{4 \gamma_{m}}}, \\
C_{2, m, 0}(x)=-3 \xi^{2}\left[(x-1)^{2} \cos \omega_{m} x-\frac{1}{\omega_{m}}(x-1) \sin \omega_{m} x\right] \\
+\frac{\left(1+\varkappa \omega_{m}^{2}\right)^{2}}{\omega_{m}}\left[\sigma_{m}\left(2 \lambda_{m, 2}+1\right)-i \lambda_{m, 1}^{2}\left(1+4 \varkappa \omega_{m}^{2}\right)\right] B_{m}(x) .
\end{gathered}
$$

В случае коэффициентов $C_{2, m, 2}^{ \pm}$сталкиваемся с той же ситуацией, что и в п. 2.1: при выполнении условий (2.45) они определяются однозначно посредством формул (2.44), в которых теперь $\xi$ - амплитуда (3.4).

На следующем шаге ситуация примерно повторяется. А именно, $w_{3, m}$ имеет ту же структуру, что и $w_{2, m}$, причем разрешимости краевой задачи для коэффициента при нулевой гармонике в этой функции добиваемся за счет выбора $\lambda_{m, 2}$. Опуская вполне понятные выкладки, отметим лишь равенство

$$
\lambda_{m, 2}=-\frac{1}{2}-\frac{9 i \varkappa \xi^{2} \sigma_{m}}{\left(1+\varkappa \omega_{m}^{2}\right)^{2}} .
$$

Из (3.26), (3.27) заключаем, что действительные части интересующих нас характеристических показателей отрицательны и имеют порядок $\varepsilon$. Таким образом, казалось бы нет смысла в проведении еще одного шага алгоритма и в нахождении $w_{4, m}$. Однако, как вытекает из проделанного в п. 2.2 анализа, при строгом обосновании свойств устойчивости задачи (3.9) необходимо знать ее приближенные решения Ляпунова-Флоке (3.10), (3.22) с точностью до $\varepsilon^{2}$ включительно.

Итак, обратимся к краевой задаче (3.23) при $j=4$ и заметим, что ее решение следует искать в виде тригонометрического полинома переменной $\sigma_{n} \tau$, содержащего только четные гармоники до четвертого порядка. Далее, разрешимость краевой задачи для коэффициента при нулевой гармонике обеспечивается выбором $\lambda_{m, 3}$. Что же касается всех остальных коэффициентов из $w_{4, m}$, то при выполнении условий (2.45) и аналогичных условий нерезонансности

$$
\begin{gathered}
\left(\sigma_{m} \pm 4 \sigma_{n}\right)^{2}-\sigma_{k}^{2} \neq 0, \quad\left(\sigma_{m} \pm 4 \sigma_{n}\right)^{2}-\frac{1}{\varkappa} \neq 0, \quad\left(\frac{1}{\sqrt{\varkappa}} \pm 4 \sigma_{n}\right)^{2}-\sigma_{k}^{2} \neq 0, \\
\left(\frac{1}{\sqrt{\varkappa}} \pm 4 \sigma_{n}\right)^{2}-\frac{1}{\varkappa} \neq 0, \quad m \in \mathbb{N}, \quad k \in \mathbb{N}, \quad m \neq n, \quad
\end{gathered}
$$

они определяются однозначно. 
Завершая описание алгоритмической части, остановимся на причине, по которой в (3.22) введена еще одна постоянная $\lambda_{m, 4}$. Ситуация здесь та же, что и в п. 2.1. А именно, за счет выбора $\lambda_{m, 4}$ мы "убиваем" неограниченно растущие при $m \rightarrow \infty$ коэффициенты в $w_{4, m}$, что, в свою очередь, обеспечивает корректность бесконечномерной замены переменных вида (2.58), (2.59), строящейся на основе решений (3.10), (3.22).

Обоснование всех изложенных алгоритмов проводится в полном соответствии с разработанной в п. 2.2 схемой. На этом пути получаем следующее утверждение, являющееся аналогом теоремы 2.1 .

ТЕОРема 3.1. Предположим, что при некотором натуральном $n$ выполняются условия (2.20), (2.45), (3.8), (3.28). Тогда при всех достаточно мальх $\varepsilon>0$ краевая задача (3.1) имеет экспоненциально орбитально устойчивый иикл с асимптотикой (3.2), (3.4).

Сформулированная теорема гарантирует реализуемость в краевой задаче (3.1) явления, которое можно назвать развитой буферностъю: для любого натурального $N$ существует такое $\varepsilon_{N}>0$, что при всех $0<\varepsilon \leqslant \varepsilon_{N}$ и при некоторой общности положения, связанной с параметром $\varkappa$, данная задача имеет не менее $N$ устойчивых циклов с частотами, близкими к $\sigma_{n}, n=1, \ldots, N$, и амплитудами (3.4). Для пояснения термина "развитая буферность" отметим, что в рамках задачи (1.27) видно, как каждый из циклов, подрастая по амплитуде, становится устойчивым, т.е. просматривается механизм возникновения феномена буферности. В случае же краевой задачи (3.1) ситуация стабилизировалась: все упомянутые выше циклы обрели устойчивость и, более того, выравнялись по амплитудам.

3.2. Заключение. Подводя итог, отметим, что полученные в этой статье результаты закрывают поставленный в [5] вопрос о теоретическом объяснении ряда экспериментов, относящихся к $R C L$-автогенераторам с отрезком соленоида в цепи обратной связи. Стало понятно, что буферность в рассматриваемом классе генераторов представляет собой универсальный феномен, не зависящий от геометрии линии (соленоида, дискретной реализации и т.д.). Более того, при переходе от классической длинной линии к соленоиду диапазон изменения коэффициента усиления, в котором наблюдается буферность, заведомо расширяется. Действительно, как следует из результатов статьи [5], при $\varkappa=0$ в краевой задаче (3.1) имеют место разрывные колебания, а при $\varkappa>0$ происходит их сглаживание и наступает развитая буферность.

В связи с вышесказанным представляет интерес оценка количества сосуществующих устойчивых циклов у краевой задачи (3.1) при фиксированных $\gamma$, $\varkappa>0$ и при $\varepsilon \rightarrow 0$. Из теорем $2.1,3.1$ следует, что устойчивыми будут циклы с частотами $\sigma_{n}$, номера которых удовлетворяют неравенству

$$
\frac{\gamma}{\varepsilon}>2 \gamma_{n}-\gamma_{1}
$$

где $\gamma_{n}$ - критические значения (1.33). Количество же таких номеров неограниченно увеличивается при $\varepsilon \rightarrow 0$, а точнее говоря, имеет порядок $\varepsilon^{-1 / 6}$.

Среди проблем, оставшихся без рассмотрения в настоящей работе, отметим вопрос об автоколебаниях краевой задачи (1.27) при фиксированном $\gamma>1 / 8$ 
и при существенном уменьшении параметра $\varkappa$, например, при $\varkappa=\varkappa_{0} \varepsilon, \varkappa_{0}=$ const $>0$. Из теоремы 2.1 косвенным образом следует, что в этом случае также должно происходить неограниченное увеличение числа сосуществующих устойчивых циклов. Строгое же исследование данного вопроса в связи с резонансностью собственных частот, имеющей место при $\varepsilon=0, \varkappa=0$, требует привлечения метода бесконечномерной нормализации в том его варианте, который описан в п. 2.3 .

\section{Список литературы}

[1] А. А. Витт, "Распределенные автоколебательные системы", ЖТФ, 4:1 (1934), 146157.

[2] А. Ю. Колесов, Е. Ф. Мищенко, Н. Х. Розов, Асимптотические методы исследования периодических решений нелинейных гиперболических уравнений, Тр. МИАН, 222, Наука, М., 1998.

[3] А. Ю. Колесов, Н.Х. Розов, В.Г. Сушко, "Специфика автоколебательных процессов в резонансных гиперболических системах", Фундам. и прикл. матем., 5:2 (1999), 437-473.

[4] А. Ю. Колесов, Е. Ф. Мищенко, Н. Х. Розов, "Явление буферности в резонансных системах гиперболических уравнений", УМН, 55:2 (2000), 95-120.

[5] А. Ю. Колесов, Н.Х. Розов, "Явление буферности в $R C L G$-автогенераторе: теоретический анализ и результаты эксперимента", Дифференциалъные уравнения. Некоторые математические задачи оптимального управления, Тр. МИАН, 233, Наука, М., 2001, 153-207.

[6] Л. В. Постников, В. И. Королев (ред.), Сборник задач по теории колебаний, Наука, М., 1978.

[7] М. И. Рабинович, Д. И. Трубецков, Введение в теорию колебаний и волн, Наука, M., 1984.

[8] Ю. С. Колесов, "Математическая теория RC-автогенераторов с распределенными параметрами в цепи обратной связи", Дифференииальные уравнения и их применение, № 2, Ин-т физики и математики АН ЛитССР, Вильнюс, 1971, 1-67.

[9] А. Ю. Колесов, Н.Х. Розов, Инвариантные торы нелинейных волновых уравнений, Физматлит, М., 2004.

[10] Л. П. Шильников, А. Л. Шильников, Д. В. Тураев, Л. Чуа, Методы качественной теории в нелинейной динамике, ч. 1, Ин-т компьютерных исследований, М., Ижевск, 2004.

[11] А. Б. Васильева, С. А. Кащенко, Ю. С. Колесов, Н. Х. Розов, "Бифуркация автоколебаний нелинейных параболических уравнений с малой диффузией", Матем. сб., 130:4 (1986), 488-499.

[12] Ю. С. Колесов, "Бифуркация инвариантных торов параболических систем с малой диффузией", Матем. сб., 184:3 (1993), 121-136.

\section{А. Ю. Колесов (А. Yu. Kolesov)}

Ярославский государственный университет

им. П. Г. Демидова

E-mail: kolesov@uniyar.ac.ru

\section{Н. Х. Розов (N. Kh. Rozov)}

Московский государственный университет

им. М. В. Ломоносова

E-mail: rozov@rozov.mccme.ru
Поступила в редакцию 14.02.2005 